\section{Arkivoc

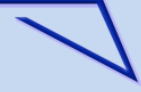

Archive for

Organic Chemistry
The Free Internet Journal

for Organic Chemistry
Paper

Arkivoc 2018, part iii, 20-35

\title{
Synthesis of quinazolindionyl amino acid and hydrazone derivatives as possible antitumour agents
}

\author{
A. Aboelmagd, ${ }^{* a}$ Ezzeldin M. S. Salem, ${ }^{a}$ Ibrahim A. I. Ali, ${ }^{a}$ and Mohamed S. Gomaab \\ aDepartment of Chemistry, Faculty of Science, Suez Canal University, Ismailia, 41522, Egypt \\ ${ }^{b}$ Department of Medicinal Chemistry, Faculty of Pharmacy, Suez Canal University, Ismailia, 41522, Egypt \\ Email: ahmedaelmagd@gmail.com
}

Received 08-23-2017

Accepted 10-22-2017

Published on line 11-26-2017

\section{Abstract}

A series of 1-ethyl-2,4-dioxo-(1H,3H)-quinazolin-3-yl amino acid and hydrazone derivatives were synthesized and tested for their antitumor activity. The alcohol and acid derivatives of quinazolindione were conjugated with the amino acid derivatives at N-3 site via ester or amide bonds by carbodiimide and azide methods. The carbodiimide-mediated amide and esterification steps were performed in the presence of HOBt or DMAP respectively otherwise the side-products $\mathrm{N}$-acyl urea derivatives are formed, instead of the desired derivatives. Nine compounds exhibited encouraging antitumor activity against human liver carcinoma cell line (HepG2).

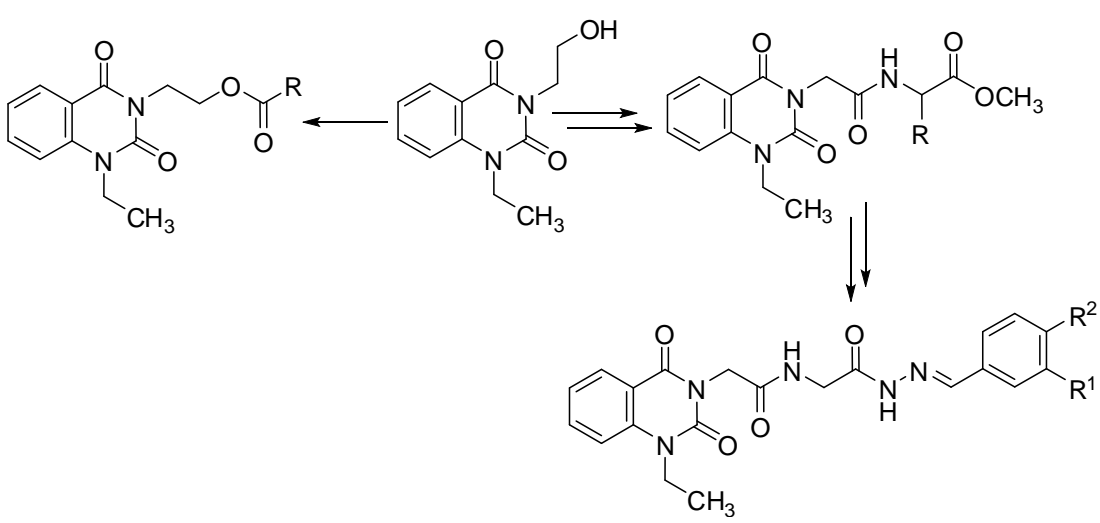

Keywords: Amino acids, quinazolindione, carbodiimide and azide coupling methods, hydrazones, antitumour activity 


\section{Introduction}

Cancer is one of the major causes of death in the developed nations. ${ }^{1-3}$ Chemotherapy with cytotoxic drugs is one of the main approaches to dealing with established cancer. ${ }^{4,5}$ The main drawbacks of the current chemotherapy of cancer are the severe toxic effects such as emesis and myelosuppression, in addition to the lack of selectivity of the drugs against tumour cells as compared with normal cells. ${ }^{1,6}$ Hence, search for newer anticancer drugs is never-ending task. Quinazolines are one of the most studied moieties in cancer chemotherapy. ${ }^{7-13}$ Recently, FDA has approved several quinazoline derivatives as antitumour drugs from past 15 years such as gefitinib, erlotinib, lapatinib and raltitrexed. ${ }^{14-18}$ It should be noted that a group of peptideantibiotics such as actinomycin D (Dactinomycin) and bleomycin has been reported as antitumour agents. ${ }^{19,20}$ Moreover, in literature it is established that anticancer effects have been shown with various hydrazones. ${ }^{21-23}$ Procarbazine, $\mathrm{N}$-isopropyl-4-[(2-methylhydrazino)methyl]-benzamide, is one of the antitumour compounds used in clinical practice. ${ }^{24}$

Based upon the afore-mentioned data, the present manuscript deals with the synthesis of a series of novel compounds containing quinazolindione moiety conjugated with amino acid and hydrazone residues, to evaluate their antitumour action. The amino acids used are selected to contain different physiologically active side-chains such as hydroxyl, phenolic, heterocyclic, sulphur-containing and alkyl groups (serine, tyrosine, tryptophan methionine, glycine, and leucine respectively). The synthesized derivatives include esters, free acid, hydrazides and hydrazones (Schemes 1-3). The newly synthesized derivatives were screened for their antitumour activity against liver carcinoma cell line (HepG2) (Tables 1).

\section{Results and Discussion}

The key compound 3-(2-hydroxyethyl)-2,4-dioxo(1H,3H)quinazoline $\mathbf{1}^{25}$ is a suitable scaffold for conjugation with amino acid derivatives at N-3 position via amide and ester bonds (Schemes 1-3). Prior to derivatization of $\mathbf{1}$, the reactive $\mathrm{N}-1$ site was protected by alkylation with ethyl iodide to yield the $\mathrm{N}-1$ ethyl derivative 2 to avoid the possible side-reactions that might occur.

Attachment of amino acids to quinazoline nucleus via amide bond required conversion of hydroxyethyl derivative $\mathbf{2}$ to the corresponding acid $\mathbf{3}$ and ester $\mathbf{4}$ (Scheme 1 ).

Accordingly, compound $\mathbf{2}$ was oxidized with alkaline potassium permanganate to afford the corresponding free acid 3. Esterification of 3 either with $\mathrm{CH}_{3} \mathrm{OH} / \mathrm{SOCl}_{2}$ or with $\left(\mathrm{CH}_{3}\right)_{2} \mathrm{SO}_{4} / \mathrm{K}_{2} \mathrm{CO}_{3}$ yielded the methyl ester 4 in $64 \%$ and $69 \%$ yields respectively. ${ }^{1} \mathrm{H}$ and ${ }^{13} \mathrm{C}$ NMR spectra of acid 3 and ester 4 exhibited the presence of their respective hydrogens and carbons in their expected trivial positions. Hydrazide $\mathbf{5}$ was prepared in $80 \%$ yield by boiling the methyl ester 4 in methanol with six-fold excess hydrazine hydrate. The ${ }^{1} \mathrm{H} N M R$ spectrum of hydrazide derivative 5 showed new signals characteristic to the hydrazide group at 9.25 and $4.21 \mathrm{ppm}$ of $\mathrm{NH}$ and $\mathrm{NH}_{2}$ respectively.

The glycine methyl ester derivative $6 \mathbf{6}$ was synthesized by two different routes i.e. the azide and carbodiimide methods. In the first procedure, the azide obtained from hydrazide $\mathbf{5}$ by treatment with nitrous acid at $0{ }^{\circ} \mathrm{C}$, reacted directly without isolation, with glycine methyl ester to afford the quinazolindione glycine methyl ester derivative $6 a$ in $47 \%$ yield. The reaction was carried out at low temperature to avoid Curtius rearrangement of the azide to the corresponding isocyanate. ${ }^{26}$ On the other hand, when the acid $\mathbf{3}$ was coupled with glycine methyl ester hydrochloride in the presence of dicyclohexyl-carbodiimide DCC, and triethyl amine in acetonitrile the isolated product was 1-(1-ethyl-2,4-dioxo-(1H,3H)-quinazolin-3-ylacetyl)-1,3- 
dicyclohexyl urea (7) instead of desired glycine methyl ester derivative 6a. The structure of the by-product $\mathrm{N}$ acyl urea derivative 7 was elucidated by ${ }^{1} \mathrm{H} N M R,{ }^{13} \mathrm{C}$ NMR and elemental analysis. However, pure sample of the desired derivative 6 a was obtained solely in a relatively higher yield (59\%) when the reaction was repeated in the presence of DCC / HOBt. The additive HOBt was used to suppress the formation of the by-product Nacyl urea derivative $7 .^{27}$<smiles>CCn1c(=O)n(CCO)c(=O)c2ccc(CCO)cc21</smiles><smiles>[R]C(NC(=O)Cn1c(=O)c2ccccc2n(CC)c1=O)C(=O)OCC1CC1CC1CC1</smiles><smiles>CCCC</smiles>

6a: Gly $\mathrm{R}=-\mathrm{H}$ 6b: $\operatorname{Ser} \mathrm{R}=-\mathrm{CH}_{2} \mathrm{OH}$ 6c: $\operatorname{Trp} \mathrm{R}=-\mathrm{CH}_{2}$<smiles>Cc1c[nH]c2ccccc12</smiles>

6d: Met R $=-\mathrm{CH}_{2} \mathrm{CH}_{2} \mathrm{SCH}_{3}$ 6e: Leu $\mathrm{R}=-\mathrm{CH}_{2} \mathrm{CH}\left(\mathrm{CH}_{3}\right)_{2}$ 6f: Tyr $\mathrm{R}=-\mathrm{CH}_{2}-\mathrm{OH}$<smiles>CC(=O)CN(C)C(=O)c1ccccc1</smiles><smiles>CC(C)N(C(=O)Cn1c(=O)c2ccccc2n(C)c1=O)C(=O)NC1CCCCC1</smiles>
$\mathrm{H}_{3} \mathrm{C}-7$<smiles>C/C=C/N(CC)C(=O)O</smiles>

5

Scheme 1. Synthesis of 1-ethyl-2,4-dioxo-(1H,3H)-quinazolin-3-ylacetyl amino acid derivatives. Reagent and conditions: a) Etl, anhydrous $\mathrm{K}_{2} \mathrm{CO}_{3}, \mathrm{DMSO}, 90{ }^{\circ} \mathrm{C}, 4 \mathrm{~h}$; b) (i) $\mathrm{KMnO}_{4}, \mathrm{Na}_{2} \mathrm{CO}_{3}$, water $70{ }^{\circ} \mathrm{C}, 6 \mathrm{~h}$; (ii) Conc. $\mathrm{HCl}$ to $\mathrm{pH} 3$; c) $\mathrm{MeOH}, \mathrm{SOCl}_{2}$, r.t., $24 \mathrm{~h}$; d) dimethyl sulfate, anhydrous $\mathrm{K}_{2} \mathrm{CO}_{3}$, acetone, reflux $5 \mathrm{~h}$; e) $\mathrm{N}_{2} \mathrm{H}_{4} . \mathrm{H}_{2} \mathrm{O}, \mathrm{MeOH}$, reflux $6 \mathrm{~h}$; f) $\mathrm{NaNO}_{2} / \mathrm{HCl}, \mathrm{AcOH},-5{ }^{\circ} \mathrm{C}$, stirring $0.5 \mathrm{~h}$; g) $\mathrm{HCl} \cdot \mathrm{H}_{2} \mathrm{NCH}_{2} \mathrm{CO}_{2} \mathrm{CH}_{3}, \mathrm{Et}{ }_{3} \mathrm{~N}$, in ethyl acetate, in refrigerator $12 \mathrm{~h}$ and at r.t. $12 \mathrm{~h}$; h) $\mathrm{HCl} . \mathrm{H}_{2} \mathrm{NCHRCO}_{2} \mathrm{CH}_{3}, \mathrm{Et}_{3} \mathrm{~N}, \mathrm{DCC}$ in $\mathrm{MeCN}, 0{ }^{\circ} \mathrm{C} 2 \mathrm{~h}$ and at r.t. overnight; i) $\mathrm{HCl} . \mathrm{H}_{2} \mathrm{NCHRCO}_{2} \mathrm{CH}_{3}, \mathrm{Et}_{3} \mathrm{~N}, \mathrm{DCC}, \mathrm{HOBt}$ in $\mathrm{MeCN}, \mathrm{O}^{\circ} \mathrm{C} 2 \mathrm{~h}$ and at r.t. overnight.

Accordingly, the remaining amino acid methyl ester derivatives $6 \mathbf{b}-\mathbf{f}$ were prepared by coupling the acid $\mathbf{3}$ with the amino acid methyl esters (L-Ser-OMe, L-Trp-OMe, L-Met-OMe, L-Leu-OMe, and L-Tyr-OMe respectively) by DCC / HOBt activation procedure in 45-56\% yield. The chemical structures of 6a-f were assigned by elemental analyses, ${ }^{1} \mathrm{H}$ and ${ }^{13} \mathrm{C}$ NMR. The ${ }^{1} \mathrm{H}$ NMR spectra of amino acid derivatives $6 a-f$ exhibited 
the following common data: multiplet signals between 6.85-6.35 ppm are of the NH protons of the peptide bonds, multiplet signals between 4.97-4.11 ppm of the $\alpha-\mathrm{CH}$ protons of the amino acids and singlet signals in the range 3.80-3.65 ppm for the three protons of the ester $\mathrm{OCH}_{3}$ groups. ${ }^{1} \mathrm{H}$ and ${ }^{13} \mathrm{C} \mathrm{NMR}$ signals for protons and carbons of each side chain of the amino acids and quinazoline ring are reported in the experimental part.

Prior to the synthesis of the physiologically important hydrazones $\mathbf{1 0 a - d}$, the hydrazide $\mathbf{9}$ was prepared by hydrazinolysis of ester 6a (Scheme 2). We report herein, the synthesis of a series of quinazolindione glycyl hydrazone derivatives $\mathbf{1 0 a - d}$ by condensation of the corresponding hydrazide $\mathbf{9}$ with some aromatic aldehydes (benzaldehyde, anisaldehyde, 3-nitrobenzaldehyde and vanillin).
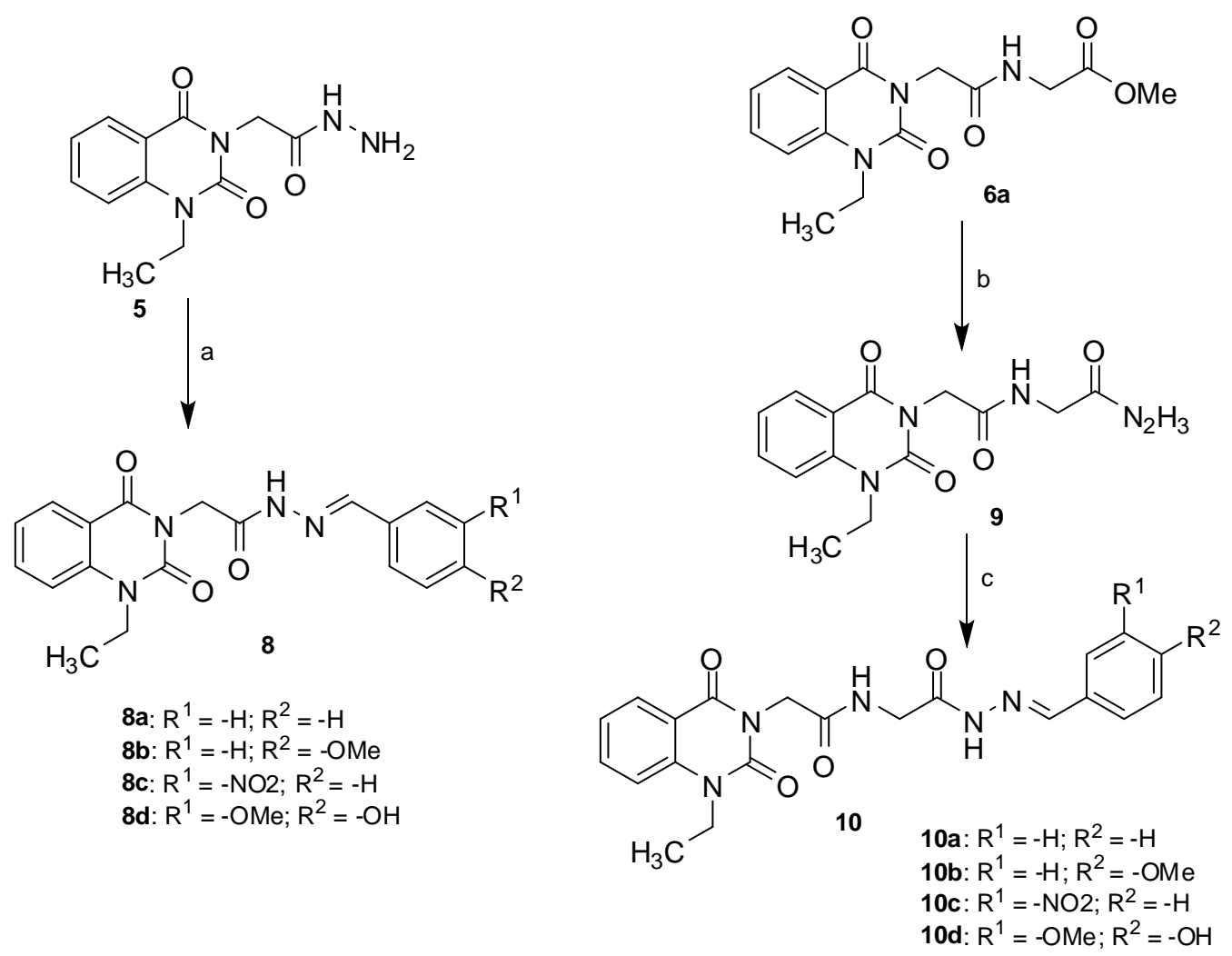

Scheme 2. Synthesis of 1-ethyl-2,4-dioxo-(1H,3H)-quinazolin-3-ylacetyl hydrazone derivatives. Reagent and conditions: a) $\mathrm{ArCHO}$, EtOH, reflux, 3 h; b) $\mathrm{N}_{2} \mathrm{H}_{4} \cdot \mathrm{H}_{2} \mathrm{O}, \mathrm{MeOH}$, reflux, 8 h; c) ArCHO, EtOH, reflux, 5 h.

On the other hand, the corresponding hydrazones, but without glycine, 8a-d have been analogously also prepared from hydrazide 5 to study the effect of the amino acid on the antitumour potency. The ${ }^{1} \mathrm{H} N M R$ of the synthesized hydrazones (in DMSO- $d_{6}$ ) showed that some protons exhibit two sets of signals for each proton $\left(\mathrm{NCH}_{2} \mathrm{CO}\right.$ and $-\mathrm{N}=\mathrm{CH}$ of the hydrazones $8 \mathrm{a}-\mathrm{d}$ and $-\mathrm{NH}-\mathrm{N}$, peptide $\mathrm{NH},-\mathrm{N}=\mathrm{CH}$, and $\alpha-\mathrm{CH}_{2}$ of the hydrazone derivatives $\mathbf{1 0 a - d}$ ), which reveals the existence of an equilibrium mixture from cis/trans amide conformers of the $E$-form (see experimental part). This conclusion is supported by previously published data on similar compounds. ${ }^{28-31}$

As has been mentioned above, this study deals also with the synthesis of some amino acid quinazolindionyl ethyl ester derivatives (Scheme 3). Surprisingly enough, trials to esterify phthalylglycine with the alcohol derivative $\mathbf{2}$ with DCC in the presence of HOBt failed to give the desired ester $\mathbf{1 2}$ and instead, the by-product $N$-phthalylglycyl dicyclohexyl urea $\mathbf{1 1}$ was obtained. However, the alcohol $\mathbf{2}$ underwent successful acylation when reacted with acetic acid, benzoic acid or the sterically hindered phthalylglycine in the presence 
of DCC / DMAP to afford the corresponding esters 13a, 13b and 12 respectively. The structures of 11, 12, 13a and $\mathbf{1 3 b}$ were confirmed by ${ }^{1} \mathrm{H} N M R,{ }^{13} \mathrm{C} N M R$, and elemental analyses (see the experimental part). Moreover, the structures of $\mathbf{1 3 a}$ and $\mathbf{1 3 b}$ were further established by their synthesis by the classical methods i.e. by acylation of $\mathbf{2}$ with acetic anhydride or benzoyl chloride in pyridine respectively. From these test experiments, it seems that DMAP is superior additive to HOBt in DCC-mediated esterification of such type of compounds.

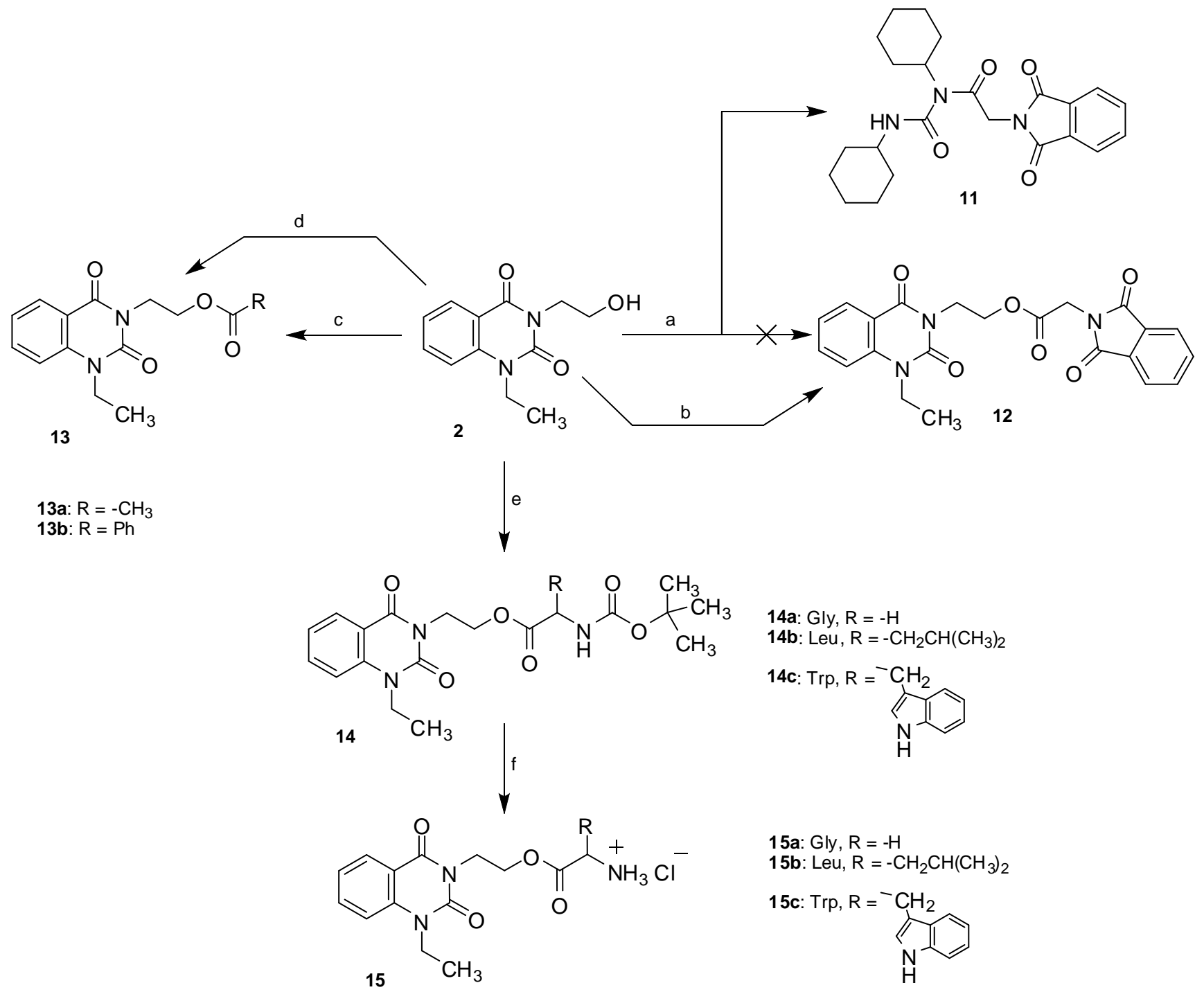

Scheme 3. Synthesis of amino acid 2-(1-ethyl-2,4-dioxo-(1H,3H)-quinazolin-3-yl)ethyl ester derivatives. Reagent and conditions: a) phth-Gly, DCC, $\mathrm{HOBt}, \mathrm{CH}_{2} \mathrm{Cl}_{2}$, r.t. 3 h; b) phth-Gly, DCC, DMAP, $\mathrm{CH}_{2} \mathrm{Cl}_{2}$ r.t. 3 h; c) $\mathrm{AcOH}$ or $\mathrm{PhCOOH}, \mathrm{DCC}$, DMAP, $\mathrm{CH}_{2} \mathrm{Cl}_{2}$, r.t. $3 \mathrm{~h}$; d) $\mathrm{AC}_{2} \mathrm{O}$ or $\mathrm{PhCOCl}$, pyridine, $0^{\circ} \mathrm{C}, 1 \mathrm{~h}$; e) Boc-amino acid, DCC, DMAP, $\mathrm{CH}_{2} \mathrm{Cl}_{2}$, r.t. $3 \mathrm{~h}$; f) $1 \mathrm{M} \mathrm{HCl}$ in ethyl acetate, r.t. $2 \mathrm{~h}$.

Accordingly, $t$-butyloxycarbonyl-amino acid quinazolinyl ethyl esters $14 a-b$ have been prepared in $63-67 \%$ yields by acylation of the alcohol 2, with the corresponding Boc-amino acid (Gly, Leu, Trp) in the presence of DCC / DMAP. The ${ }^{1} \mathrm{H}$ NMR spectra of these derivatives showed a common singlet signal in the range between 1.39-1.26 ppm corresponding to the nine protons of the tert-butyloxy group $-\mathrm{OC}\left(\mathrm{CH}_{3}\right)_{3}$ and a broad signal between 5.24-5.01 ppm attributed to the proton of the Boc-NH group. ${ }^{13} \mathrm{C}$ NMR spectra displayed the characteristic signals between 79.9-79.2 ppm of the tert-carbon $-\mathrm{OC}\left(\mathrm{CH}_{3}\right)_{3}$ and between 28.2-27.8 ppm of the 
carbons of the three methyl groups $-\mathrm{OC}\left(\mathrm{CH}_{3}\right)_{3}$, in addition to other signals corresponding to protons and carbons of the different side chains of the amino acids and quinazoline ring which add further support to the structures of the prepared esters (refer to the experimental part).

The Boc-groups were cleaved by the action of $1 \mathrm{M} \mathrm{HCl}$ in ethyl acetate to yield the corresponding hydrochloride salts $15 \mathrm{a}-\mathrm{c}$ in $44-74 \%$ yield. ${ }^{1} \mathrm{H}$ NMR and ${ }^{13} \mathrm{C}$ NMR spectra of these compounds revealed the disappearance of the characteristic signals of the Boc protons and carbons and instead the presence of broad signals between 8.90-8.20 ppm for the three protons of $\mathrm{NH}_{3}{ }^{+}$group, alongwith other signals for protons and carbons of the individual side chains of the corresponding amino acids (refer to the experimental part).

The synthesized quinazolindione derivatives were tested for their antiproliferative activity against human liver carcinoma cell line (HepG2) where they showed promising activity (Table 1).

Table 1. In vitro antiproliferative activity of 1-ethyl-2,4-dioxo-(1H,3H)-quinazolin-3-ylacetyl amino acid and hydrazone derivatives against human liver carcinoma cell line (HepG2)

\begin{tabular}{|c|c|c|c|c|c|}
\hline \multirow[t]{3}{*}{ Compd. } & \multicolumn{4}{|c|}{$\begin{array}{l}\text { Compound concentration } \\
\qquad(\mu \mathrm{g} / \mathrm{mL})\end{array}$} & \multirow{3}{*}{$\begin{array}{c}\mathrm{IC}_{50} \\
(\mu \mathrm{g} / \mathrm{mL})\end{array}$} \\
\hline & 12.5 & 25 & 50 & 100 & \\
\hline & \multicolumn{4}{|c|}{ Surviving fraction } & \\
\hline 1 & 1.000 & 0.922 & 0.682 & 0.622 & $>100$ \\
\hline 2 & 0.729 & 0.521 & 0.370 & 0.344 & 28.00 \\
\hline 5 & 0.572 & 0.420 & 0.352 & 0.300 & 18.30 \\
\hline $6 b$ & 0.724 & 0.510 & 0.464 & 0.443 & 29.30 \\
\hline $6 c$ & 1.00 & 0.954 & 0.919 & 0.910 & $>100$ \\
\hline $6 d$ & 0.441 & 0.218 & 0.165 & 0.160 & 9.68 \\
\hline $6 e$ & 0.650 & 0.541 & 0.414 & 0.391 & 33.00 \\
\hline $6 f$ & 0.792 & 0.781 & 0.681 & 0.673 & $>100$ \\
\hline 7 & 0.977 & 0.881 & 0.858 & 0.731 & $>100$ \\
\hline $8 a$ & 0.850 & 0.575 & 0.355 & 0.350 & 33.70 \\
\hline $8 d$ & 0.759 & 0.271 & 0.254 & 0.336 & 19.10 \\
\hline 9 & 1.000 & 0.696 & 0.546 & 0.380 & $>50$ \\
\hline $10 \mathrm{~b}$ & 0.714 & 0.621 & 0.514 & 0.460 & $>50$ \\
\hline 12 & 0.593 & 0.436 & 0.263 & 0.305 & 19.70 \\
\hline $13 a$ & 0.886 & 0.750 & 0.741 & 0.727 & $>100$ \\
\hline $13 b$ & 0.746 & 0.654 & 0.638 & 0.400 & $>50$ \\
\hline $14 a$ & 0.986 & 0.772 & 0.707 & 0.630 & $>100$ \\
\hline $14 b$ & 0.500 & 0.455 & 0.341 & 0.386 & 12.50 \\
\hline $14 c$ & 0.923 & 0.712 & 0.692 & 0.420 & $>50$ \\
\hline $15 a$ & 0.747 & 0.316 & 0.255 & 0.254 & 19.40 \\
\hline $15 b$ & 0.578 & 0.194 & 0.215 & 0.252 & 15.10 \\
\hline $15 c$ & 0.864 & 0.823 & 0.782 & 0.741 & $>100$ \\
\hline doxorubicin & 0.248 & 0.275 & 0.360 & 0.344 & 3.23 \\
\hline
\end{tabular}

It is clear that out of twenty seven screened novel synthesized compounds, nine compounds exhibited $\mathrm{IC}_{50}$ 's in the range of $9.68-33.7 \mu \mathrm{g} / \mathrm{mL}$ compared to the reference drug doxorubicin $\left(\mathrm{IC} \mathrm{C}_{50} 3.23 \mu \mathrm{g} / \mathrm{mL}\right.$ ). The 
methionine methyl ester derivative $\mathbf{6} \mathbf{d}$ was found to be the most active synthesized compound with $\mathrm{IC}_{50} 9.68$ $\mu \mathrm{g} / \mathrm{mL}$. this derivative $\mathbf{6 d}$ could be considered as a lead compound for further optimization and development.

\section{Conclusions}

We hereby present the synthesis and preliminary antiproliferative activity study of a series of 1-ethyl-2,4dioxo-(1H,3H)-quinazolin-3-yl amino acid derivatives. The quinazolindione nucleus was connected with amino acids by amide or ester bonds at N-3 site. The carbodiimide-mediated amide and esterification steps should be performed in the presence of HOBt or DMAP respectively.

Since the quinazolindione amino acid methyl ester derivatives were obtained in the present study with moderate yields by the azide or carbodiimide methods, other coupling procedures should be attempted with the aim to get better yields. The synthesized compounds showed good activity against HepG2 cell line and the results generated compound $6 \mathbf{d}\left(I C_{50} 9.6 \mu \mathrm{g} / \mathrm{mL}\right)$ as a lead compound for further optimization and development. Based up on the obtained promising anticancer results, it is necessary to extend this study for the synthesis of a series of N3-quinazolindione amino acid esters bearing other different open side-chains. Moreover, the synthesis of other hydrazones derived from aliphatic carbonyl compounds could afford novel derivatives with enhanced anticancer action.

\section{Experimental Section}

General. Thin layer chromatography (TLC) was carried out on silica gel $60 \mathrm{~F}_{254}$ aluminium sheets (E. Merck, layer thickness $0.2 \mathrm{~mm}$ ) in the following solvent systems, $S_{1}$ : chloroform / methanol (95:5); $S_{2}$ : chloroform/methanol (9:1); $S_{3}$ : ethyl acetate/petroleum ether (2:1); $S_{4}$ : ethyl acetate/petroleum ether (1:1). The spots on thin layer plates were detected by UV lamp. Melting points were determined on a Buchi 510 melting-point apparatus. Elemental analyses were performed on a Flash EA-1112 instrument at the Microanalytical Laboratory, Faculty of Science, Suez Canal University, Ismailia, Egypt. ${ }^{1} \mathrm{H}$ and ${ }^{13} \mathrm{C}$ NMR spectra were measured on Bruker spectrometer operating at 300 and $75 \mathrm{MHz}$ respectively, at microanalytical laboratory, Cairo University, Giza, Egypt. The starting compounds 3-(2-hyd-roxyethyl)-2,4-di-oxo-(1H,3H)quinazoline 1 and 1-ethyl-3-(2-hydroxyeth-yl)-2,4-dioxo-(1H,3H)-quinazoline $\mathbf{2}$ were prepared according to the described method in literature. ${ }^{25}$ Potential cytotoxicity of the newly synthesized compounds was tested against human liver carcinoma cell line (HepG2) at pharmacology unit in cancer biology department at the National Cancer Institute, Cairo University, Cairo.

1-Ethyl-2,4-dioxo-(1H,3H)-quinazolin-3-ylacetic acid (3). To a suspension of 2 (2.34 g, $10 \mathrm{mmol}$ ) in sodium carbonate solution ( $1.05 \mathrm{~g}$, in $25 \mathrm{~mL}$ water) $\mathrm{KMnO}_{4}$ solution ( $4.0 \mathrm{~g}$ in $25 \mathrm{~mL}$ water) was added. The reaction mixture was heated on water bath at $70{ }^{\circ} \mathrm{C}$ for $6 \mathrm{hrs}$. Afterwards, the $\mathrm{MnO}_{2}$ was filtered off and the cold filtrate was acidified with conc. $\mathrm{HCl}$ to $\mathrm{pH} 3$. The formed white ppt, after filtration, was washed with cold water, and crystallized from aqueous ethanol to yield white crystals ( $1.81 \mathrm{~g}, 73 \%), R_{f}=0.53\left(S_{2}\right), m p 227-229^{\circ} \mathrm{C}$. ${ }^{1} \mathrm{H}$ NMR (300 MHz, DMSO-d $\left.)_{6}\right): \delta 13.00(1 \mathrm{H}, \mathrm{brs}, \mathrm{OH}), 8.08(1 \mathrm{H}, \mathrm{d}, J 9.1 \mathrm{~Hz}, \mathrm{ArH}), 7.84-7.78(1 \mathrm{H}, \mathrm{t}, J 9.1 \mathrm{~Hz}, \mathrm{ArH})$, $7.56(1 \mathrm{H}, \mathrm{d}, J 9.1 \mathrm{~Hz}, \mathrm{ArH}), 7.35-7.30(1 \mathrm{H}, \mathrm{t}, J 9.1 \mathrm{~Hz}, \mathrm{ArH}), 4.62\left(2 \mathrm{H}, \mathrm{s}, \mathrm{NCH}_{2} \mathrm{CO}\right), 4.19-4.12\left(2 \mathrm{H}, \mathrm{m}, \mathrm{NCH}_{2} \mathrm{CH}_{3}\right)$, 1.24-1.15 (3H, m, NCH $\left.\mathrm{CH}_{3}\right) .{ }^{13} \mathrm{C} \mathrm{NMR}\left(75 \mathrm{MHz}, \mathrm{DMSO}-d_{6}\right): \delta 169.2,160.7,149.62(3 \mathrm{CO}), 139.2,135.7,128.1$, 
122.8, 114.6, 114.4 (Ar-C), $42.3\left(\mathrm{NCH}_{2} \mathrm{CO}\right), 38.3\left(\mathrm{NCH}_{2} \mathrm{CH}_{3}\right), 12.3\left(\mathrm{NCH}_{2} \mathrm{CH}_{3}\right)$. Anal. Calcd. For $\mathrm{C}_{12} \mathrm{H}_{12} \mathrm{~N}_{2} \mathrm{O}_{4}$ (248.23): C, 58.06; H, 4.87; N, 11.29; Found C, 58.16; H, 4.82; N, 11.44.

\section{Methyl 1-ethyl-2,4-dioxo-(1H,3H)-quinazolin-3-ylacetate (4)}

Method A. To a suspension of $3(2.48 \mathrm{~g}, 10 \mathrm{mmol})$ in methanol $(25 \mathrm{~mL})$, thionyl chloride $(0.8 \mathrm{~mL}, 11 \mathrm{mmol}) \mathrm{was}$ added dropwise. The reaction mixture was stirred at room temperature for $24 \mathrm{hrs}$. Afterwards, the methanol and excess thionyl chloride were evaporated under reduced pressure. The residue was dissolved in methanol $(10 \mathrm{~mL})$ and evaporated under reduced pressure to get rid of residual thionyl chloride. Finally, the residue was crystallized from ethyl acetate /petroleum ether to yield 4 as white crystals $(1.69 \mathrm{~g}, 64 \%) . \mathrm{R}_{\mathrm{f}}=0.64\left(\mathrm{~S}_{3}\right)$, $\mathrm{mp} 84-$ $86^{\circ} \mathrm{C}$.

Method B. A mixture of $3(2.48 \mathrm{~g}, 10 \mathrm{mmol})$, anhydrous potassium carbonate $(1.37 \mathrm{~g})$ and methyl sulphate $(0.95 \mathrm{~mL}, 10 \mathrm{mmol})$ in acetone $(25 \mathrm{~mL})$ was heated under reflux for $5 \mathrm{hrs}$. The reaction mixture was poured under stirring into ice-water mixture, and then the solid precipitate was filtered, washed with water and dried in air. The crude product was crystallized from ethyl acetate /petroleum ether to yield $\mathbf{4}$ as white crystals (1.81 g, 69\%). $\mathrm{R}_{\mathrm{f}}=0.64\left(\mathrm{~S}_{3}\right), \mathrm{mp} 83-86^{\circ} \mathrm{C} .{ }^{1} \mathrm{H} \mathrm{NMR}\left(300 \mathrm{MHz}, \mathrm{CDCl}_{3}\right): \delta 8.24(1 \mathrm{H}, \mathrm{d}, J 7.8 \mathrm{~Hz}, \mathrm{ArH}), 7.72-7.66(1 \mathrm{H}, \mathrm{t}, J$ 7.8 Hz, ArH), 7.28-7.22 (2H, m, ArH), $4.85\left(2 \mathrm{H}, \mathrm{s}, \mathrm{NCH}_{2}\right), 4.23-4.16\left(2 \mathrm{H}, \mathrm{m}, \mathrm{NCH}_{2} \mathrm{CH}_{3}\right), 3.77(3 \mathrm{H}, \mathrm{s}, \mathrm{OCH}), 1.37-$ $1.33\left(3 \mathrm{H}, \mathrm{t}, J 7.2 \mathrm{~Hz}, \mathrm{NCH}_{2} \mathrm{CH}_{3}\right) .{ }^{13} \mathrm{C} \mathrm{NMR}\left(75 \mathrm{MHz}, \mathrm{CDCl}_{3}\right): \delta 168.4,161.3,150.1$ (3CO), 139.6, 135.4, 129.3, 122.9, 115.4, $113.5(\mathrm{Ar}-\mathrm{C}), 52.4\left(\mathrm{OCH}_{3}\right), 42.3\left(\mathrm{NCH}_{2}\right), 38.9\left(\mathrm{NCH}_{2}\right), 12.5\left(\mathrm{CH}_{3}\right)$. Anal. Calcd. For $\mathrm{C}_{13} \mathrm{H}_{14} \mathrm{~N}_{2} \mathrm{O}_{4}$ (262.26): C, 59.54; H, 5.38; N, 10.68; Found C, 59.87; H, 5.58; N, 10.93.

1-Ethyl-2,4-dioxo-(1H,3H)-quinazolin-3-ylacetyl hydrazide (5). To a solution of 4 (2.0 $\mathrm{g}, 7.62 \mathrm{mmol}) \mathrm{in}$ methanol $(30 \mathrm{ml})$, hydrazine hydrate $(3 \mathrm{ml}, 48.0 \mathrm{mmol})$ was added. The reaction mixture was refluxed for 6 hrs, after cooling to room temperature the precipitated hydrazide was filtered off, washed with water and ethanol followed by recrystallization from aqueous ethanol to give 5 as white crystals $(1.61 \mathrm{~g}, 80 \%), R_{f}=$ 0.52( $\left(\mathrm{S}_{2}\right), \mathrm{mp} 218-221{ }^{\circ} \mathrm{C} .{ }^{1} \mathrm{H}$ NMR $\left(300 \mathrm{MHz}, \mathrm{DMSO}-d_{6}\right): \delta 9.25(1 \mathrm{H}, \mathrm{s}, \mathrm{NH}), 8.07(1 \mathrm{H}, \mathrm{d}, J 7.5 \mathrm{~Hz}, \mathrm{ArH}), 7.82-7.76$ $(1 \mathrm{H}, \mathrm{t}, J 7.5 \mathrm{~Hz}, \mathrm{ArH}), 7.53(1 \mathrm{H}, \mathrm{d}, J 7.5 \mathrm{~Hz}, \mathrm{ArH}), 7.33-7.28(1 \mathrm{H}, \mathrm{t}, J 7.5 \mathrm{~Hz}, \mathrm{ArH}), 4.51\left(2 \mathrm{H}, \mathrm{s}, \mathrm{NCH}_{2}\right), 4.21(2 \mathrm{H}, \mathrm{s}$, $\left.\mathrm{NH}_{2}\right), 4.17-4.10\left(2 \mathrm{H}, \mathrm{m}, \mathrm{NCH}_{2} \mathrm{CH}_{3}\right), 1.24-1.19\left(3 \mathrm{H}, \mathrm{t}, J 7.0 \mathrm{~Hz}, \mathrm{NCH}_{2} \mathrm{CH}_{3}\right) .{ }^{13} \mathrm{C} \mathrm{NMR}\left(75 \mathrm{MHz}, \mathrm{DMSO}-d_{6}\right): \delta 166.7$, 161.4, 150.3 (3CO), 139.9, 135.9, 128.5, 123.1, 115.5, $114.8(\mathrm{Ar}-\mathrm{C}), 42.8\left(\mathrm{NCH}_{2}\right), 38.7\left(\mathrm{NCH}_{2}\right), 12.8\left(\mathrm{CH}_{3}\right)$. Anal. Calcd. For $\mathrm{C}_{12} \mathrm{H}_{14} \mathrm{~N}_{4} \mathrm{O}_{3}$ (262.26): C, 54.96; $\mathrm{H}, 5.38 ; \mathrm{N}, 21.36$; Found C, 54.98; $\mathrm{H}, 5.67 ; \mathrm{N}, 21.10$.

\section{1-Ethyl-2,4-dioxo-(1H,3H)-quinazolin-3-ylacetyl glycine methyl ester (6a)}

Method A. Azide coupling method: To a cold solution $\left(-5^{\circ} \mathrm{C}\right)$ of hydrazide $5(0.21 \mathrm{~g}, 0.8 \mathrm{mmol})$ in acetic acid (6 $\mathrm{ml})$, hydrochloric acid $(5 \mathrm{~N}, 3 \mathrm{ml})$, and water $(25 \mathrm{ml})$, was added portionwise under stirring a cold solution $\left(0^{\circ} \mathrm{C}\right)$ of sodium nitrite $(0.07 \mathrm{~g}, 1.0 \mathrm{mmol})$ in water $(3 \mathrm{ml})$. After stirring at the same temperature for 30 minutes, the azide was extracted with cold ethyl acetate, and washed successively with cold water, $5 \% \mathrm{NaHCO}_{3}$ and water. After drying over anhydrous sodium sulphate, the azide was used without further purification in the next step. Glycine methyl ester hydrochloride $(0.11 \mathrm{~g}, 0.9 \mathrm{mmol})$, was stirred in ethyl acetate $(30 \mathrm{~mL})$ with triethyl amine $(0.2 \mathrm{ml})$ at $0{ }^{\circ} \mathrm{C}$ for 20 minutes. The formed triethyl amine hydrochloride was filtered off and the filtrate was added to the previously prepared cold dried solution of the azide. Afterwards the mixture was kept $12 \mathrm{hrs}$ in the refrigerator and then at room temperature for another $12 \mathrm{hrs}$. The reaction mixture was washed with $0.1 \mathrm{~N}$ $\mathrm{HCl}$, water, $5 \% \mathrm{NaHCO}_{3}$ and water then dried over anhydrous sodium sulphate. The solvent was evaporated in vacuum and the residue was crystallized from ethyl acetate-petroleum ether to give 6 a as white crystals (0.12 g, 47\%) $R_{f}=0.23\left(S_{3}\right), m p ~ 199-202^{\circ} \mathrm{C}$.

Method B. DCC coupling method: To a cold solution $\left(-5^{\circ} \mathrm{C}\right)$ of glycine methyl ester hydrochloride $(0.55 \mathrm{~g}, 4.4$ $\mathrm{mmol})$ and triethyl amine $(0.62 \mathrm{ml} .4 .4 \mathrm{mmol})$ in acetonitrile $(40 \mathrm{ml})$ 1-ethyl-2,4-dioxo-(1H,3H)-quinazolin-3ylacetic acid (3) (1.09 g, $4.4 \mathrm{mmol})$, HOBt (0.6 g, $4.4 \mathrm{mmol})$, and DCC (0.92 g, $4.4 \mathrm{mmol})$ were added successively. The reaction mixture was stirred at $0{ }^{\circ} \mathrm{C}$ for $2 \mathrm{hrs}$. and at room temperature overnight. The 
precipitated dicyclohexylurea (DCU) was filtered off and the filtrate was evaporated under reduced pressure. The residue was extracted with ethyl acetate, filtered off from the remaining DCU, afterwards the filtrate was washed successively with saturated $\mathrm{NaCl}$ solution, $5 \% \mathrm{NaHCO}_{3}$ solution, $1 \mathrm{M} \mathrm{HCl}$ and water. After drying over anhydrous $\mathrm{Na}_{2} \mathrm{SO}_{4}$, the solvent was evaporated to dryness and the remaining residue was crystallized from ethyl acetate/petroleum ether to yield 1-ethyl-2,4-dioxo-(1H,3H)-quinazolin-3-ylacetyl glycine methyl ester (6a) as white crystals (0.82 g, 58\%), $\mathrm{R}_{\mathrm{f}}=0.23\left(\mathrm{~S}_{3}\right), \mathrm{mp} 201-203{ }^{\circ} \mathrm{C} .{ }^{1} \mathrm{H}$ NMR $\left(300 \mathrm{MHz}, \mathrm{CDCl}_{3}\right): \delta 8.24(1 \mathrm{H}, \mathrm{d}, J 7.8$ $\mathrm{Hz}, \mathrm{ArH}), 7.71-7.65(1 \mathrm{H}, \mathrm{t}, \mathrm{J} 7.8 \mathrm{~Hz}, \mathrm{ArH}), 7.27-7.21(2 \mathrm{H}, \mathrm{m}, \mathrm{ArH}), 6.42(1 \mathrm{H}, \mathrm{brs}, \mathrm{NH}), 4.83\left(2 \mathrm{H}, \mathrm{s}, \mathrm{NCH}_{2}\right), 4.24-$ $4.17\left(2 \mathrm{H}, \mathrm{m}, \mathrm{NCH}_{2} \mathrm{CH}_{3}\right), 4.11\left(2 \mathrm{H}, \mathrm{d}, J 5.1 \mathrm{~Hz}, \mathrm{NCH}_{2}\right), 3.75\left(3 \mathrm{H}, \mathrm{s}, \mathrm{OCH}_{3}\right), 1.38-1.33\left(3 \mathrm{H}, \mathrm{t}, J 7.2 \mathrm{~Hz}, \mathrm{NCH}_{2} \mathrm{CH}_{3}\right) .{ }^{13} \mathrm{C}$ NMR (75 MHz, CDCl $)$ ): $\delta$ 170.1, 167.0, 161.6, 150.3 (4CO), 139.7, 135.3, 129.4, 122.9, 115.5, 113.5 (Ar-C), 52.4 $\left(\mathrm{OCH}_{3}\right), 44.0\left(\mathrm{NCH}_{2} \mathrm{CO}\right), 41.3\left(\mathrm{NCH}_{2} \mathrm{CO}\right), 39.0\left(\mathrm{NCH}_{2} \mathrm{CH}_{3}\right), 12.5\left(\mathrm{CH}_{3}\right)$. Anal. Calcd. For $\mathrm{C}_{15} \mathrm{H}_{17} \mathrm{~N}_{3} \mathrm{O}_{5}$ (319.31): C, 56.42; H, 5.37; N, 13.16; Found C, 56.57; H, 5.43; N, 13.26.

1-(1-Ethyl-2,4-dioxo-(1H,3H)-quinazolin-3-ylacetyl)-1,3-dicyclohexyl urea (7). To a cold solution $\left(-5{ }^{\circ} \mathrm{C}\right)$ of glycine methyl ester hydrochloride $(0.55 \mathrm{~g}, 4.4 \mathrm{mmol})$ and triethyl amine $(0.62 \mathrm{ml} .4 .4 \mathrm{mmol})$ in acetonitrile (40 $\mathrm{ml}$ ) 1-ethyl-2,4-dioxo-(1H,3H)-quinazolin-3-ylacetic acid (3) (1.09 g, $4.4 \mathrm{mmol})$, and DCC (0.92 g, $4.4 \mathrm{mmol})$ were added successively. The reaction mixture was stirred at $0{ }^{\circ} \mathrm{C}$ for $2 \mathrm{hrs}$. and at room temperature overnight. The precipitated DCU was filtered off and the filtrate was evaporated under reduced pressure. The residue was extracted with ethyl acetate, filtered off from the remaining DCU, afterwards the filtrate was washed successively with saturated $\mathrm{NaCl}$ solution, $5 \% \mathrm{NaHCO}_{3}$ solution, $1 \mathrm{M} \mathrm{HCl}$ and water. After drying over anhydrous $\mathrm{Na}_{2} \mathrm{SO}_{4}$, the solvent was evaporated to dryness and the remaining residue was crystallized from ethyl acetate/petroleum ether to yield 1-(1-ethyl-2,4-dioxo-(1H,3H)-quinazolin-3-ylacetyl)-1,3-dicyclohexyl urea (7) as white crystals (1.43 g, 71\%), $\mathrm{R}_{\mathrm{f}}=0.87\left(\mathrm{~S}_{2}\right), \mathrm{mp} 155-158{ }^{\circ} \mathrm{C} .{ }^{1} \mathrm{H} \mathrm{NMR}\left(300 \mathrm{MHz}, \mathrm{CDCl}_{3}\right): \delta 8.24(1 \mathrm{H}, \mathrm{d}, J$ $7.8 \mathrm{~Hz}, \mathrm{ArH}), 7.72-7.67(1 \mathrm{H}, \mathrm{t}, J 7.8 \mathrm{~Hz}, \mathrm{ArH}), 7.42(1 \mathrm{H}, \mathrm{d}, J 6.3 \mathrm{~Hz}, \mathrm{NH}), 7.29-7.20(2 \mathrm{H}, \mathrm{m}, \operatorname{ArH}), 4.99(2 \mathrm{H}, \mathrm{s}$, $\left.\mathrm{NCH}_{2}\right), 4.24-4.17\left(2 \mathrm{H}, \mathrm{m}, \mathrm{NCH}_{2} \mathrm{CH}_{3}\right), 4.10-3.95(1 \mathrm{H}, \mathrm{m}, \mathrm{NCH}), 3.80-3.62(1 \mathrm{H}, \mathrm{m}, \mathrm{NCH}), 2.01-1.15\left(23 \mathrm{H}, \mathrm{m}, 10 \mathrm{CH}_{2}\right.$ cyclohexyl rings, $\left.\mathrm{NCH}_{2} \mathrm{CH}_{3}\right) .{ }^{13} \mathrm{C} \mathrm{NMR}\left(75 \mathrm{MHz}, \mathrm{CDCl}_{3}\right): \delta 166.9,161.7,153.3,150.4(4 \mathrm{CO}), 139.7,135.4,129.3$, 122.9, 115.4, 113.5 (Ar-C), 56.4, 49.9 (2NCH-cyclohexyl rings), $44.0\left(\mathrm{NCH}_{2} \mathrm{CO}\right), 39.0\left(\mathrm{NCH}_{2} \mathrm{CH}_{3}\right), 32.5,30.6,26.3$, 25.4, 25.2, 24.7 ( $\mathrm{CH}_{2}$-cyclohexyl rings), $12.5\left(\mathrm{NCH}_{2} \mathrm{CH}_{3}\right)$. Anal. Calcd. For $\mathrm{C}_{25} \mathrm{H}_{34} \mathrm{~N}_{4} \mathrm{O}_{4}$ (454.56): C, 66.06; $\mathrm{H}, 7.54$; $\mathrm{N}, 12.33$; Found $\mathrm{C}, 66.19 ; \mathrm{H}, 7.57 ; \mathrm{N}, 12.51$.

1-Ethyl-2,4-dioxo-(1H,3H)-quinazolin-3-ylacetyl amino acid methyl esters (6b-f). General method. To a cold solution $\left(-5^{\circ} \mathrm{C}\right)$ of amino acid methyl ester hydrochloride $(4.4 \mathrm{mmol})$ and triethyl amine $(0.62 \mathrm{ml} .4 .4 \mathrm{mmol})$ in acetonitrile $(40 \mathrm{ml})$ acid derivative $3(1.09 \mathrm{~g}, 4.4 \mathrm{mmol})$, HOBt $(0.6 \mathrm{~g}, 4.4 \mathrm{mmol})$, and DCC (0.92 g, $4.4 \mathrm{mmol})$ were added successively. The reaction mixture was treated as described above under the synthesis of the glycine methyl ester derivative $6 \mathbf{a}$ (Method B).

1-Ethyl-2,4-dioxo-(1H,3H)-quinazolin-3-ylacetyl L-serine methyl ester (7b). White crystals $(0.68 \mathrm{~g}, 45 \%), \mathrm{R}_{\mathrm{f}}=$ 0.15(S $\left.\mathrm{S}_{3}\right), \mathrm{mp} 213-216{ }^{\circ} \mathrm{C} .{ }^{1} \mathrm{H}$ NMR $\left(300 \mathrm{MHz}, \mathrm{CDCl}_{3}\right): \delta 8.20(1 \mathrm{H}, \mathrm{d}, J 7.8 \mathrm{~Hz}, \mathrm{ArH}), 7.68-7.63(1 \mathrm{H}, \mathrm{t}, J 7.9 \mathrm{~Hz}$, $\operatorname{ArH}), 7.26-7.19(3 \mathrm{H}, \mathrm{m}, \mathrm{ArH}, \mathrm{NH}), 4.90\left(1 \mathrm{H}, \mathrm{d}, J_{\text {gem }} 15.3 \mathrm{~Hz}, \mathrm{NCHCO}\right), 4.78\left(1 \mathrm{H}, \mathrm{d}, J_{\text {gem }} 15.3 \mathrm{~Hz}, \mathrm{NCHCO}\right), 4.65-$ $4.60(1 \mathrm{H}, \mathrm{m}, \mathrm{NCH}), 4.340-4.25(1 \mathrm{H}, \mathrm{brs}, \mathrm{OH}), 4.20-4.13\left(2 \mathrm{H}, \mathrm{m}, \mathrm{NCH}_{2} \mathrm{CH}_{3}\right), 3.93-3.85\left(2 \mathrm{H}, \mathrm{m}, \mathrm{CH}_{2} \mathrm{O}\right), 3.74(3 \mathrm{H}, \mathrm{s}$, $\left.\mathrm{OCH}_{3}\right), 1.34-1.29\left(3 \mathrm{H}, \mathrm{t}, J 7.2 \mathrm{~Hz}, \mathrm{NCH}_{2} \mathrm{CH}_{3}\right) .{ }^{13} \mathrm{C} \mathrm{NMR}\left(75 \mathrm{MHz}, \mathrm{CDCl}_{3}\right): \delta$ 170.7, 167.0, 160.7, 147.0 (4CO), 139.6, 136.6, 129.2, 122.7, 115.1, $113.4(\mathrm{Ar}-\mathrm{C}), 62.7\left(\mathrm{OCH}_{2}\right), 54.9(\mathrm{NCH}), 52.4\left(\mathrm{OCH}_{3}\right), 43.9\left(\mathrm{NCH}_{2} \mathrm{CO}\right), 38.8$ $\left(\mathrm{NCH}_{2} \mathrm{CH}_{3}\right), 12.4\left(\mathrm{CH}_{3}\right)$. Anal. Calcd. For $\mathrm{C}_{16} \mathrm{H}_{19} \mathrm{~N}_{3} \mathrm{O}_{6}$ (349.34): C, 55.01; $\mathrm{H}, 5.48 ; \mathrm{N}, 12.03$; Found C, 55.19; $\mathrm{H}$, $5.45 ; \mathrm{N}, 12.22$.

1-Ethyl-2,4-dioxo-(1H,3H)-quinazolin-3-ylacetyl L-tryptophane methyl ester (6c). White crystals (1.11 g, 56\%), $\mathrm{R}_{\mathrm{f}}=0.36\left(\mathrm{~S}_{3}\right), \mathrm{mp} 118-120{ }^{\circ} \mathrm{C} .{ }^{1} \mathrm{H} \mathrm{NMR}\left(300 \mathrm{MHz}, \mathrm{CDCl}_{3}\right): \delta 8.43(1 \mathrm{H}, \mathrm{brs}$, Ind-NH), $8.24(1 \mathrm{H}, \mathrm{d}, J 7.8 \mathrm{~Hz}, \mathrm{ArH})$, 7.67- $7.65(1 \mathrm{H}, \mathrm{m}, \mathrm{ArH}), 7.53(1 \mathrm{H}, \mathrm{d}, \mathrm{J} 7.8 \mathrm{~Hz}, \mathrm{ArH}), 7.28-7.05(6 \mathrm{H}, \mathrm{m}, 5 \mathrm{ArH}, \mathrm{Ind}-2-\mathrm{H}), 6.54-6.80(1 \mathrm{H}, \mathrm{d}, J 8.1 \mathrm{~Hz}$, $\mathrm{NH}), 4.97-4.94(1 \mathrm{H}, \mathrm{m}, \mathrm{NCH}), 4.82\left(1 \mathrm{H}, \mathrm{d}, J_{\text {gem }} 15.3 \mathrm{~Hz}, \mathrm{NCHCO}\right), 4.72\left(1 \mathrm{H}, \mathrm{d}, J_{\text {gem }} 15.3 \mathrm{~Hz}, \mathrm{NCHCO}\right), 4.17-4.10$ 
$\left(2 \mathrm{H}, \mathrm{m}, \mathrm{NCH}_{2} \mathrm{CH}_{3}\right), 3.65\left(3 \mathrm{H}, \mathrm{s}, \mathrm{OCH}_{3}\right), 3.34\left(2 \mathrm{H}, \mathrm{d}, \mathrm{CH}_{2}\right), 1.34-1.29\left(3 \mathrm{H}, \mathrm{t}, J 7.1 \mathrm{~Hz}, \mathrm{NCH}_{2} \mathrm{CH}_{3}\right) .{ }^{13} \mathrm{C} \mathrm{NMR}(75 \mathrm{MHz}$, $\left.\mathrm{CDCl}_{3}\right): \delta 172.1,166.8,161.6,150.3$ (4CO), 139.6, 136.1, 135.2, 129.2, 127.5, 123.6, 122.8, 121.7, 119.3, 118.3, 115.4, 113.5, 111.2, $109.1(\mathrm{Ar}-\mathrm{C}), 53.2(\mathrm{NCH}), 52.3\left(\mathrm{OCH}_{3}\right), 43.8\left(\mathrm{NCH}_{2} \mathrm{CO}\right), 38.9\left(\mathrm{NCH}_{2} \mathrm{CH}_{3}\right), 27.4\left(\mathrm{CH}_{2}\right), 12.5$ $\left(\mathrm{CH}_{3}\right)$. Anal. Calcd. For $\mathrm{C}_{24} \mathrm{H}_{24} \mathrm{~N}_{4} \mathrm{O}_{5}$ (448.47): C, 64.28; $\mathrm{H}, 5.39 ; \mathrm{N}, 12.49$; Found $\mathrm{C}, 64.56 ; \mathrm{H}, 5.68 ; \mathrm{N}, 12.28$.

Ethyl-2,4-dioxo-(1H,3H)-quinazolin-3-ylacetyl L-methionine methyl ester (6d). White crystals $(0.88 \mathrm{~g}, 51 \%), \mathrm{R}_{\mathrm{f}}$ $=0.48\left(\mathrm{~S}_{3}\right), \mathrm{mp} 168-170{ }^{\circ} \mathrm{C} .{ }^{1} \mathrm{H} \mathrm{NMR}\left(300 \mathrm{MHz}, \mathrm{CDCl}_{3}\right): \delta 8.25(1 \mathrm{H}, \mathrm{d}, J 7.8 \mathrm{~Hz}, \mathrm{ArH}), 7.71-7.66(1 \mathrm{H}, \mathrm{t}, J 7.8 \mathrm{~Hz}$, $\operatorname{ArH})$, 7.28-7.21 (2H, m, ArH), $6.54(1 \mathrm{H}, \mathrm{d}, J 7.5 \mathrm{~Hz}, \mathrm{NH}), 4.90\left(1 \mathrm{H}, \mathrm{d}, J_{\text {gem }} 15.6 \mathrm{~Hz}, \mathrm{NCHCO}\right), 4.76\left(1 \mathrm{H}, \mathrm{d}, J_{\text {gem }} 15.6\right.$ $\mathrm{Hz}, \mathrm{NCHCO}), 4.76-4.71(1 \mathrm{H}, \mathrm{m}, \mathrm{NCH}), 4.24-4.17\left(2 \mathrm{H}, \mathrm{m}, \mathrm{NCH}_{2} \mathrm{CH}_{3}\right), 3.76\left(3 \mathrm{H}, \mathrm{s}, \mathrm{OCH}_{3}\right), 2.56-2.51(2 \mathrm{H}, \mathrm{m}, \mathrm{SCH})$, 2.19-2.00 (2H, m, CH 2$), 2.07\left(3 \mathrm{H}, \mathrm{s}, \mathrm{SCH}_{3}\right), 1.36-1.32\left(3 \mathrm{H}, \mathrm{t}, J 7.1 \mathrm{~Hz}, \mathrm{NCH}_{2} \mathrm{CH}_{3}\right) .{ }^{13} \mathrm{C} \mathrm{NMR}\left(75 \mathrm{MHz}, \mathrm{CDCl}_{3}\right): \delta$ 172.1, 166.7, 161.5, 150.3 (4CO), 139.6, 135.3, 129.4, 122.9, 115.5, 113.5 (Ar-C), $52.4\left(\mathrm{OCH}_{3}\right), 51.7$ (NCH), 44.1 $\left(\mathrm{NCH}_{2} \mathrm{CO}\right), 39.0\left(\mathrm{NCH}_{2} \mathrm{CH}_{3}\right), 31.7\left(\mathrm{SCH}_{2}\right), 29.8\left(\mathrm{CH}_{2}\right), 15.4\left(\mathrm{SCH}_{3}\right), 12.5\left(\mathrm{CH}_{3}\right)$. Anal. Calcd. For $\mathrm{C}_{18} \mathrm{H}_{23} \mathrm{~N}_{3} \mathrm{O}_{5} \mathrm{~S}$ (393.46): C, 54.95; H, 5.89; N, 10.68; S, 8.15; Found C, 55.19; H, 6.04; N, 10.41; S, 8.38.

Ethyl-2,4-dioxo-(1H,3H)-quinazolin-3-ylacetyl L-leucine methyl ester (6e). White crystals $(0.78 \mathrm{~g}, 47 \%), \mathrm{R}_{\mathrm{f}}=$ $0.54\left(\mathrm{~S}_{3}\right), \mathrm{mp} 141-144{ }^{\circ} \mathrm{C} .{ }^{1} \mathrm{H} \mathrm{NMR}\left(300 \mathrm{MHz}, \mathrm{CDCl}_{3}\right): \delta 8.23(1 \mathrm{H}, \mathrm{d}, J 8.1 \mathrm{~Hz}, \mathrm{ArH}), 7.69-7.64(1 \mathrm{H}, \mathrm{t}, J 8.1 \mathrm{~Hz}, \mathrm{ArH})$, 7.26-7.20 (2H, m, ArH), $6.36(1 \mathrm{H}, \mathrm{d}, J 7.8 \mathrm{~Hz}, \mathrm{NH}), 4.86\left(1 \mathrm{H}, \mathrm{d}, J_{\text {gem }} 15.3 \mathrm{~Hz}, \mathrm{NCHCO}\right), 4.78\left(1 \mathrm{H}, \mathrm{d}, J_{\text {gem }} 15.3 \mathrm{~Hz}\right.$, $\mathrm{NCHCO})$, 4.71-4.64 (1H, m, NCH), 4.22-4.15 $\left(2 \mathrm{H}, \mathrm{m}, \mathrm{NCH}_{2} \mathrm{CH}_{3}\right), 3.72\left(3 \mathrm{H}, \mathrm{s}, \mathrm{OCH}_{3}\right), 1.71-1.53(3 \mathrm{H}, \mathrm{m}, \mathrm{CH}, \mathrm{CH})$, 1.36-1.32 (3H, t, J $\left.7.1 \mathrm{~Hz}, \mathrm{NCH}_{2} \mathrm{CH}_{3}\right), 0.93\left(6 \mathrm{H}, \mathrm{d}, J 6.3 \mathrm{~Hz}, 2 \mathrm{CH}_{3}\right) .{ }^{13} \mathrm{C} \mathrm{NMR}\left(75 \mathrm{MHz}, \mathrm{CDCl}_{3}\right): \delta 173.2,166.6$, 161.5, 150.3 (4CO), 139.6, 135.3, 129.3, 122.8, 115.5, $113.4(\mathrm{Ar}-\mathrm{C}), 52.2\left(\mathrm{OCH}_{3}\right), 50.9(\mathrm{NCH}), 43.9(\mathrm{NCH} 2 \mathrm{CO})$, $41.9\left(\mathrm{NCH}_{2} \mathrm{CH}_{3}\right), 38.7(\mathrm{CH}), 24.7\left(\mathrm{CH}_{2}\right), 22.6,22.04\left(2 \mathrm{CH}_{3}\right), 12.5\left(\mathrm{CH}_{3}\right)$. Anal. Calcd. For $\mathrm{C}_{19} \mathrm{H}_{25} \mathrm{~N}_{3} \mathrm{O}_{5}(375.42): \mathrm{C}$, 60.79; H, 6.71; N, 11.19; Found C, 61.02; H, 6.97; N, 11.44.

Ethyl-2,4-dioxo-(1H,3H)-quinazolin-3-ylacetyl L-tyrosine methyl ester (6f). White crystals $(0.93 \mathrm{~g}, 50 \%), \mathrm{R}_{\mathrm{f}}=$ 0.36(S $\left.\mathrm{S}_{3}\right), \mathrm{mp} 184-18{ }^{\circ} \mathrm{C} .{ }^{1} \mathrm{H}$ NMR $\left(300 \mathrm{MHz}, \mathrm{CDCl}_{3}\right): \delta 8.26(1 \mathrm{H}, \mathrm{d}, J 7.8 \mathrm{~Hz}, \mathrm{ArH}), 7.72-7.67(1 \mathrm{H}, \mathrm{t}, J 7.8 \mathrm{~Hz}$, $\operatorname{ArH}), 7.29-7.22(2 \mathrm{H}, \mathrm{m}, \mathrm{ArH}), 6.96(2 \mathrm{H}, \mathrm{d}, J 8.4 \mathrm{~Hz}, \mathrm{ArH}), 6.68(2 \mathrm{H}, \mathrm{d}, J 8.4 \mathrm{~Hz}, \mathrm{ArH}), 6.35(1 \mathrm{H}, \mathrm{d}, J 7.8 \mathrm{~Hz}, \mathrm{NH})$, 4.90-4.84 (H, m, NCH), $4.84\left(1 \mathrm{H}, \mathrm{d}, J_{\text {gem }} 15.3 \mathrm{~Hz}, \mathrm{NCHCO}\right), 4.75\left(1 \mathrm{H}, \mathrm{d}, J_{\text {gem }} 15.3 \mathrm{~Hz}, \mathrm{NCHCO}\right), 4.22-4.16(2 \mathrm{H}, \mathrm{m}$, $\left.\mathrm{NCH}_{2} \mathrm{CH}_{3}\right), 3.73\left(3 \mathrm{H}, \mathrm{s}, \mathrm{OCH}_{3}\right), 3.09-3.05\left(2 \mathrm{H}, \mathrm{m}, \mathrm{CH}_{2} \mathrm{Ph}\right), 1.35-1.26\left(3 \mathrm{H}, \mathrm{t}, J 7.2 \mathrm{~Hz}, \mathrm{NCH}_{2} \mathrm{CH}_{3}\right) .{ }^{13} \mathrm{C} \mathrm{NMR}(75 \mathrm{MHz}$, $\mathrm{CDCl}_{3}$ ): $\delta 171.8,166.6,161.6$ (3CO), 155.5 (Ar-C) 150.3 (CO), 139.6, 135.4, 130.4, 129.3, 126.7, 122.9, 115.5, $113.4(\mathrm{Ar}-\mathrm{C}), 52.3\left(\mathrm{OCH}_{3}\right), 49.1(\mathrm{NCH}), 44.0\left(\mathrm{NCH}_{2} \mathrm{CO}\right), 39.0\left(\mathrm{NCH}_{2} \mathrm{CH}_{3}\right), 25.5\left(\mathrm{CH}_{2}\right), 12.4\left(\mathrm{CH}_{3}\right)$. Anal. Calcd. For $\mathrm{C}_{22} \mathrm{H}_{23} \mathrm{~N}_{3} \mathrm{O}_{6}$ (425.43): C, 62.11; $\mathrm{H}, 5.45 ; \mathrm{N}, 9.88$; Found $\mathrm{C}, 62.30 ; \mathrm{H}, 5.72 ; \mathrm{N}, 10.21$.

General procedure for the synthesis of hydrazones (8a-d). A mixture of hydrazide $5(0.3 \mathrm{~g}, 1.14 \mathrm{mmol})$ and aromatic aldehyde $(1.2 \mathrm{mmol})$ was refluxed in ethanol $(20 \mathrm{ml})$ for 3 hours. After cooling to room temperature, the resulting solid was filtered, washed with cold ethanol and recrystallized from aqueous ethanol.

1-Ethyl-2,4-dioxo-(1H,3H)-quinazolin-3-ylacetyl benzylidene hydrazone (8a). White crystals $(0.36 \mathrm{~g}, 91 \%), \mathrm{R}_{\mathrm{f}}=$ 0.66 $\left(\mathrm{S}_{1}\right), \mathrm{mp} 274-276{ }^{\circ} \mathrm{C} .{ }^{1} \mathrm{H}$ NMR $\left(300 \mathrm{MHz}, \mathrm{DMSO}-d_{6}\right): \delta 11.67(1 \mathrm{H}, \mathrm{s}, \mathrm{NH}), 8.22,8.04(1 \mathrm{H}, 2 \mathrm{~s}, \mathrm{CH}), 8.09(1 \mathrm{H}, \mathrm{d}$, J 8.1 Hz, ArH), 7.83-7.78 (1H, t, J 7.8 Hz, ArH), 7.76-7.65 (2H, m, ArH), $7.55(1 \mathrm{H}, \mathrm{d}, J 8.4 \mathrm{~Hz}, \operatorname{ArH}), 7.50-7.39(3 \mathrm{H}$, $\mathrm{m}, \mathrm{ArH}), 7.35-7.30(1 \mathrm{H}, \mathrm{t}, J 7.5 \mathrm{~Hz}, \mathrm{ArH}), 5.10,4.70\left(2 \mathrm{H}, 2 \mathrm{~s}, \mathrm{NCH}_{2} \mathrm{CO}\right), 4.19-4.10\left(2 \mathrm{H}, \mathrm{m}, \mathrm{NCH}_{2} \mathrm{CH}_{3}\right), 1.25-1.20$ $\left(3 \mathrm{H}, \mathrm{t}, J 6.7 \mathrm{~Hz}, \mathrm{NCH}_{2} \mathrm{CH}_{3}\right) .{ }^{13} \mathrm{C} \mathrm{NMR}\left(75 \mathrm{MHz}, \mathrm{DMSO}-d_{6}\right): \delta$ 167.9, 160.8149 .7 (3CO), 144.0 (CH), 139.2, 135.6, 134.0, 129.9, 128.7, 128.1, 126.8, 122.7, 114.8, $114.3(\mathrm{Ar}-\mathrm{C}), 42.3\left(\mathrm{NCH}_{2} \mathrm{CO}\right), 38.2\left(\mathrm{NCH}_{2} \mathrm{CH}_{3}\right), 12.3\left(\mathrm{CH}_{3}\right)$. Anal. Calcd. For $\mathrm{C}_{19} \mathrm{H}_{18} \mathrm{~N}_{4} \mathrm{O}_{3}$ (350.37): C, 65.13; H, 5.18; N, 15.99; Found C, 64.87; H, 5.16; N, 16.23 .

1-Ethyl-2,4-dioxo-(1H,3H)-quinazolin-3-ylacetyl 4 -methoxybenzylidene hydrazone (8b). White crystals $(0.42$ g, 96\%), $R_{f}=0.68\left(S_{1}\right), m p ~ 267-269{ }^{\circ} \mathrm{C} .{ }^{1} \mathrm{H} \mathrm{NMR}\left(300 \mathrm{MHz}, \mathrm{DMSO}-d_{6}\right): \delta 11.53(1 \mathrm{H}, \mathrm{s}, \mathrm{NH}), 8.09(1 \mathrm{H}, \mathrm{d}, J 8.1 \mathrm{~Hz}$, $\operatorname{ArH}), 8.15,7.98(1 \mathrm{H}, 2 \mathrm{~s}, \mathrm{CH}), 7.83-7.78(1 \mathrm{H}, \mathrm{t}, J 7.9 \mathrm{~Hz}, \mathrm{ArH}), 7.66(1 \mathrm{H}, \mathrm{d}, J 8.7 \mathrm{~Hz}, \operatorname{ArH}), 7.55(1 \mathrm{H}, \mathrm{d}, J 8.7 \mathrm{~Hz}$, $\mathrm{ArH})$, 7.34-7.29 (1H, t, J 7.6 Hz, ArH), $7.00(1 \mathrm{H}, \mathrm{d}, J 8.7 \mathrm{~Hz}, \mathrm{ArH}), 5.07,4.68\left(2 \mathrm{H}, 2 \mathrm{~s}, \mathrm{NCH}_{2} \mathrm{CO}\right), 4.19-4.12(2 \mathrm{H}, \mathrm{m}$, $\left.\mathrm{NCH}_{2} \mathrm{CH}_{3}\right), 3.79\left(3 \mathrm{H}, \mathrm{s}, \mathrm{OCH}_{3}\right), 1.25-1.20\left(3 \mathrm{H}, \mathrm{t}, J 7.0 \mathrm{~Hz}, \mathrm{NCH}_{2} \mathrm{CH}_{3}\right) .{ }^{13} \mathrm{C}$ NMR $\left(75 \mathrm{MHz}, \mathrm{DMSO}-d_{6}\right): \delta 167.6,160.6$ 149.7 (3CO), 146.7 (Ar-C), $143.8(\mathrm{CH}), 139.2,135.5,128.3,126.5,122.7,114.8,114.2(\operatorname{Ar}-\mathrm{C}), 55.2\left(\mathrm{OCH}_{3}\right), 42.3$ 
$\left(\mathrm{NCH}_{2} \mathrm{CO}\right), 38.2\left(\mathrm{NCH}_{2} \mathrm{CH}_{3}\right), 12.3\left(\mathrm{CH}_{3}\right)$. Anal. Calcd. For $\mathrm{C}_{20} \mathrm{H}_{20} \mathrm{~N}_{4} \mathrm{O}_{4}(380.40): \mathrm{C}, 63.15 ; \mathrm{H}, 5.30 ; \mathrm{N}, 14.73 ;$ Found C, 62.91; H, 5.26; N, 14.95.

1-Ethyl-2,4-dioxo-(1H,3H)-quinazolin-3-ylacetyl $\mathbf{3}^{\backslash}$-nitrobenzylidene hydrazone (8c). Yellowish white crystals (0.37 g, 82\%), $R_{f}=0.64\left(S_{1}\right), m p ~ 280-283{ }^{\circ} \mathrm{C} .{ }^{1} \mathrm{H} N M R\left(300 \mathrm{MHz}, \mathrm{DMSO}-d_{6}\right): \delta 11.91(1 \mathrm{H}, \mathrm{s}, \mathrm{NH}), 8.47(1 \mathrm{H}, \mathrm{s}, \mathrm{ArH})$, $8.22(1 \mathrm{H}, \mathrm{d}, J 8.1 \mathrm{~Hz}, \mathrm{ArH}), 8.33,8.13(1 \mathrm{H}, 2 \mathrm{~s}, \mathrm{CH}), 8.06(1 \mathrm{H}, \mathrm{d}, J 7.5 \mathrm{~Hz}, \mathrm{ArH}), 7.82-7.76(1 \mathrm{H}, \mathrm{t}, J 7.6 \mathrm{~Hz}, \mathrm{ArH})$, 7.72-7.67 (1H, t, J 7.6 Hz, ArH), $7.53(1 \mathrm{H}, \mathrm{d}, J 8.1 \mathrm{~Hz}, \mathrm{ArH}), 7.33-7.28(1 \mathrm{H}, \mathrm{t}, J 7.4 \mathrm{~Hz}, \operatorname{ArH}), 5.12,4.73(2 \mathrm{H}, 2 \mathrm{~s}$, $\left.\mathrm{NCH}_{2} \mathrm{CO}\right), 4.19-4.12\left(2 \mathrm{H}, \mathrm{m}, \mathrm{NCH}_{2} \mathrm{CH}_{3}\right), 1.24-1.20\left(3 \mathrm{H}, \mathrm{t}, J 6.6 \mathrm{~Hz}, \mathrm{NCH}_{2} \mathrm{CH}_{3}\right) .{ }^{13} \mathrm{C} \mathrm{NMR}\left(75 \mathrm{MHz}, \mathrm{DMSO}-d_{6}\right): \delta$ 168.2, 160.8149 .6 (3CO), 148.1, 144.5 (Ar-C), $141.7(\mathrm{CH}), 139.2,135.5,132.8,130.2,128.0,124.0,122.7,120.9$ 114.6, $114.3(\mathrm{Ar}-\mathrm{C}), 42.3\left(\mathrm{NCH}_{2} \mathrm{CO}\right), 38.2\left(\mathrm{NCH}_{2} \mathrm{CH}_{3}\right), 12.3\left(\mathrm{CH}_{3}\right)$. Anal. Calcd. For $\mathrm{C}_{19} \mathrm{H}_{17} \mathrm{~N}_{5} \mathrm{O}_{5}(395.37)$ : C, 57.72; $\mathrm{H}, 4.33 ; \mathrm{N}, 17.71 ;$ Found C, 57.50; H, 4.29; N, 17.96 .

1-Ethyl-2,4-dioxo-(1H,3H)-quinazolin-3-ylacetyl 3 '-methoxy-4-hydroxybenzylidene hydrazone (8d). White crystals $(0.42 \mathrm{~g}, 94 \%), \mathrm{R}_{\mathrm{f}}=0.51\left(\mathrm{~S}_{1}\right), \mathrm{mp} 275-277{ }^{\circ} \mathrm{C} .{ }^{1} \mathrm{H}$ NMR $\left(300 \mathrm{MHz}, \mathrm{DMSO}-d_{6}\right): \delta 11.48(1 \mathrm{H}, \mathrm{s}, \mathrm{NH}), 9.48(1 \mathrm{H}$, s, OH), $8.08(1 \mathrm{H}, \mathrm{d}, J 7.8 \mathrm{~Hz}, \mathrm{ArH}), 8.10,7.92(1 \mathrm{H}, 2 \mathrm{~s}, \mathrm{CH}), 7.81-7.76(1 \mathrm{H}, \mathrm{t}, J 7.8 \mathrm{~Hz}, \operatorname{ArH}), 7.52(1 \mathrm{H}, \mathrm{d}, J 8.4 \mathrm{~Hz}$, $\operatorname{ArH}), 7.33-7.27(2 \mathrm{H}, \mathrm{m}, \mathrm{ArH}), 7.10-7.06(1 \mathrm{H}, \mathrm{t}, J 7.5 \mathrm{~Hz}, \mathrm{ArH}), 6.84(1 \mathrm{H}, \mathrm{d}, J 7.8 \mathrm{~Hz}, \mathrm{ArH}), 5.09,4.68(2 \mathrm{H}, 2 \mathrm{~s}$, $\left.\mathrm{NCH}_{2} \mathrm{CO}\right), 4.18-4.11\left(2 \mathrm{H}, \mathrm{m}, \mathrm{NCH}_{2} \mathrm{CH}_{3}\right), 3.83\left(3 \mathrm{H}, \mathrm{s}, \mathrm{OCH}_{3}\right), 1.24-1.19\left(3 \mathrm{H}, \mathrm{t}, J 6.9 \mathrm{~Hz}, \mathrm{NCH}_{2} \mathrm{CH}_{3}\right) .{ }^{13} \mathrm{C} \mathrm{NMR}(75$ $\left.\mathrm{MHz}, \mathrm{DMSO}-d_{6}\right): \delta$ 167.6, 160.9149 .7 (3CO), 148.9, $147.4(\mathrm{Ar}-\mathrm{C}), 144.4(\mathrm{CH}), 139.2,135.5,128.1,125.4,122.7$, 121.9, 121.3, 115.4, 114.3, $109.3(\mathrm{Ar}-\mathrm{C}), 55.5\left(\mathrm{OCH}_{3}\right), 42.5\left(\mathrm{NCH}_{2} \mathrm{CO}\right), 38.2\left(\mathrm{NCH}_{2} \mathrm{CH}_{3}\right), 12.3\left(\mathrm{CH}_{3}\right)$. Anal. Calcd. For $\mathrm{C}_{20} \mathrm{H}_{20} \mathrm{~N}_{4} \mathrm{O}_{5}$ (396.4): C, 60.60; $\mathrm{H}, 5.09 ; \mathrm{N}, 14.13$; Found $\mathrm{C}, 60.41 ; \mathrm{H}, 5.07 ; \mathrm{N}, 14.36$.

1-Ethyl-2,4-dioxo-(1H,3H)-quinazolin-3-ylacetyl glycine hydrazide (9). To a solution of $6 \mathrm{a}(2.0 \mathrm{~g}, 6.25 \mathrm{mmol}) \mathrm{in}$ methanol $(30 \mathrm{ml})$, hydrazine hydrate $(3 \mathrm{ml}, 48.0 \mathrm{mmol})$ was added. The reaction mixture was refluxed for 8 hrs, after cooling to room temperature the precipitated hydrazide was filtered off, washed with water, and ethanol followed by recrystallization from aqueous ethanol to give white crystals $(1.44 \mathrm{~g}, 72 \%), \mathrm{R}_{\mathrm{f}}=0.31\left(\mathrm{~S}_{2}\right)$, mp 228-231 ${ }^{\circ} \mathrm{C} .{ }^{1} \mathrm{H}$ NMR (300 MHz, DMSO-d $)$ : $\delta 8.95(1 \mathrm{H}, \mathrm{s}, \mathrm{NH}), 8.44-8.40(1 \mathrm{H}, \mathrm{m}, \mathrm{NH}), 8.07(1 \mathrm{H}, \mathrm{d}, J 7.8 \mathrm{~Hz}$, $\operatorname{ArH}), 7.83-7.78(1 \mathrm{H}, \mathrm{t}, J 7.8 \mathrm{~Hz}, \mathrm{ArH}), 7.55(1 \mathrm{H}, \mathrm{d}, J 7.8 \mathrm{~Hz}, \mathrm{ArH}), 7.34-7.29(1 \mathrm{H}, \mathrm{t}, J 7.8 \mathrm{~Hz}, \mathrm{ArH}), 4.62(2 \mathrm{H}, \mathrm{s}$, $\left.\mathrm{NCH}_{2} \mathrm{CO}\right), 4.25-4.14\left(2 \mathrm{H}\right.$, brs, $\left.\mathrm{NH}_{2}\right), 4.18-4.11\left(2 \mathrm{H}, \mathrm{m}, \mathrm{NCH}_{2} \mathrm{CH}_{3}\right), 3.70\left(2 \mathrm{H}, \mathrm{d}, J 6.0 \mathrm{~Hz}, \mathrm{NCH}_{2}\right), 1.27-1.19(3 \mathrm{H}, \mathrm{t}, J$ $\left.6.9 \mathrm{~Hz}, \mathrm{NCH}_{2} \mathrm{CH}_{3}\right) .{ }^{13} \mathrm{C} \mathrm{NMR}\left(75 \mathrm{MHz}\right.$, DMSO- $\left.d_{6}\right): \delta$ 167.9, 166.9, 160.9, 149.8 (4CO), 139.3, 135.5, 128.0, 122.6, 114.9, $114.3(\mathrm{Ar}-\mathrm{C}), 43.4\left(\mathrm{NCH}_{2}\right), 40.3\left(\mathrm{NCH}_{2} \mathrm{CO}\right), 33.2\left(\mathrm{NCH}_{2} \mathrm{CH}_{3}\right), 12.3\left(\mathrm{CH}_{3}\right)$. Anal. Calcd. For $\mathrm{C}_{14} \mathrm{H}_{17} \mathrm{~N}_{5} \mathrm{O}_{4}$ (319.32): C, 52.66; H, 5.37; N, 21.93; Found C, 52.41; H, 5.33; N, 21.87.

General procedure for the synthesis of hydrazones (10a-d). A mixture of $9(0.3 \mathrm{~g}, 0.9 \mathrm{mmol})$ and carbonyl compound $(1.0 \mathrm{mmol})$ was refluxed in ethanol $(20 \mathrm{ml})$ for 5 hours. After cooling to room temperature, the resulting solid was filtered, washed with cold ethanol and recrystallized from aqueous ethanol.

1-Ethyl-2,4-dioxo-(1H,3H)-quinazolin-3-ylacetyl glycyl benzylidene hydrazone (10a). White crystals $(0.31 \mathrm{~g}$, $79 \%), R_{f}=0.62\left(S_{2}\right) \mathrm{mp} 264-266{ }^{\circ} \mathrm{C} .{ }^{1} \mathrm{H} N M R\left(300 \mathrm{MHz}, \mathrm{DMSO}-d_{6}\right): \delta 11.46,11.30(1 \mathrm{H}, 2 \mathrm{~s}, \mathrm{NH}), 8.60-8.57,8.42-$ $8.38(1 \mathrm{H}, 2 \mathrm{~m}, \mathrm{NH}), 8.08(1 \mathrm{H}, \mathrm{d}, J 7.5 \mathrm{~Hz}, \mathrm{ArH}), 8.22,7.98(1 \mathrm{H}, 2 \mathrm{~s}, \mathrm{CH}), 7.82-7.77(1 \mathrm{H}, \mathrm{t}, J 7.5 \mathrm{~Hz}, \mathrm{ArH}), 7.69-7.29$ $(6 \mathrm{H}, \mathrm{m}, \mathrm{ArH}), 4.66\left(2 \mathrm{H}, \mathrm{s}, \mathrm{NCH}_{2} \mathrm{CO}\right), 4.30-4.28,3.86-3.80\left(2 \mathrm{H}, 2 \mathrm{~m}, \mathrm{NCH}_{2}\right), 4.19-4.12\left(2 \mathrm{H}, \mathrm{m}, \mathrm{NCH}_{2} \mathrm{CH}_{3}\right), 1.24-1.19$ $\left(3 \mathrm{H}, \mathrm{t}, J 7.1 \mathrm{~Hz}, \mathrm{NCH}_{2} \mathrm{CH}_{3}\right) .{ }^{13} \mathrm{C} \mathrm{NMR}\left(75 \mathrm{MHz}, \mathrm{DMSO}-d_{6}\right): \delta$ 170.0, 167.1, 160.9149 .7 (4CO), 146.7 (CH), 143.4, 139.3, 135.5, 134.0, 129.7, 128.7, 128.1, 126.7, 122.6, 114.8, $114.3(\mathrm{Ar}-\mathrm{C}), 43.4\left(\mathrm{NCH}_{2} \mathrm{CO}\right), 38.2\left(\mathrm{NCH}_{2} \mathrm{CH}_{3}\right)$, $12.3\left(\mathrm{CH}_{3}\right)$. Anal. Calcd. For $\mathrm{C}_{21} \mathrm{H}_{21} \mathrm{~N}_{5} \mathrm{O}_{4}$ (407.42): $\mathrm{C}, 61.91 ; \mathrm{H}, 5.20 ; \mathrm{N}, 17.19$; Found C, 62.00; H, 5.46; N, 17.47.

1-Ethyl-2,4-dioxo-(1H,3H)-quinazolin-3-ylacetyl glycyl 4-methoxybenzylidene hydrazone (10b). White crystals $(0.33 \mathrm{~g}, 82 \%), \mathrm{R}_{\mathrm{f}}=0.77\left(\mathrm{~S}_{2}\right) \mathrm{mp} 248-250{ }^{\circ} \mathrm{C} .{ }^{1} \mathrm{H}$ NMR $\left(300 \mathrm{MHz}, \mathrm{DMSO}-d_{6}\right): \delta 11.33,11.15(1 \mathrm{H}, 2 \mathrm{~s}, \mathrm{NH})$, 8.58, $8.38(1 \mathrm{H}, 2 \mathrm{~m}, \mathrm{NH}), 8.08(1 \mathrm{H}, \mathrm{d}, J 8.1 \mathrm{~Hz}, \mathrm{ArH}), 8.15,7.92(1 \mathrm{H}, 2 \mathrm{~s}, \mathrm{CH}), 7.83(1 \mathrm{H}, \mathrm{t}, J 7.5 \mathrm{~Hz}, \mathrm{ArH}), 7.65-7.52$ $(3 \mathrm{H}, \mathrm{m}, \mathrm{ArH}), 7.34(1 \mathrm{H}, \mathrm{t}, J 7.5 \mathrm{~Hz}, \mathrm{ArH}), 7.02-6.95(2 \mathrm{H}, \mathrm{m}, \mathrm{ArH}), 4.65\left(2 \mathrm{H}, \mathrm{s}, \mathrm{NCH}_{2} \mathrm{CO}\right), 4.27-4.20,4.84-3.80(2 \mathrm{H}$, $\left.2 \mathrm{~m}, \mathrm{NCH}_{2}\right), 4.19-4.12\left(2 \mathrm{H}, \mathrm{m}, \mathrm{NCH}_{2} \mathrm{CH}_{3}\right), 3.78\left(3 \mathrm{H}, \mathrm{s}, \mathrm{OCH}_{3}\right), 1.24-1.20\left(3 \mathrm{H}, \mathrm{t}, J 6.7 \mathrm{~Hz}, \mathrm{NCH}_{2} \mathrm{CH}_{3}\right) .{ }^{13} \mathrm{C} \mathrm{NMR}(75$ $\left.\mathrm{MHz}, \mathrm{DMSO}-d_{6}\right): \delta$ 170.3, 167.3, 161.3 (3CO), 161.1 (Ar-C), 150.2 (CO), 143.8 (CH), 139.8, 136.0, 129.11, 128.8, 
128.6, 127.1, 123.2, 115.4, $114.7(\mathrm{Ar}-\mathrm{C}), 55.7\left(\mathrm{OCH}_{3}\right), 43.0\left(\mathrm{NCH}_{2} \mathrm{CO}\right), 38.7\left(\mathrm{NCH}_{2} \mathrm{CH}_{3}\right), 12.8\left(\mathrm{CH}_{3}\right)$. Anal. Calcd. For $\mathrm{C}_{22} \mathrm{H}_{23} \mathrm{~N}_{5} \mathrm{O}_{5}$ (437.45): C, 60.40; $\mathrm{H}, 5.30 ; \mathrm{N}, 16.01$; Found $\mathrm{C}, 60.03 ; \mathrm{H}, 5.53 ; \mathrm{N}, 16.09$.

1-Ethyl-2,4-dioxo-(1H,3H)-quinazolin-3-ylacetyl glycyl $\mathbf{3}$-nitrobenzylidene hydrazone (10c). Yellowish white crystals $(0.27 \mathrm{~g}, 64 \%), \mathrm{R}_{\mathrm{f}}=0.66\left(\mathrm{~S}_{2}\right), \mathrm{mp} 301-304{ }^{\circ} \mathrm{C} .{ }^{1} \mathrm{H}$ NMR $\left(300 \mathrm{MHz}, \mathrm{DMSO}-d_{6}\right): \delta 11.68,11.60(1 \mathrm{H}, 2 \mathrm{~s}, \mathrm{NH})$, 8.62, 8.49 (1H, 2brs., NH), 8.49-7.27 (7H, m, ArH), 8.42, $8.08(1 \mathrm{H}, 2 \mathrm{~s}, \mathrm{CH}), 4.66\left(2 \mathrm{H}, \mathrm{s}, \mathrm{NCH}_{2} \mathrm{CO}\right), 4.35-4.30$, 3.90-3.85 (2H, $\left.2 \mathrm{~m}, \mathrm{NCH}_{2}\right), 4.15-4.12\left(2 \mathrm{H}, \mathrm{m}, \mathrm{NCH}_{2} \mathrm{CH}_{3}\right), 1.23-1.19\left(3 \mathrm{H}, \mathrm{t}, J 6.3 \mathrm{~Hz}, \mathrm{NCH}_{2} \mathrm{CH}_{3}\right) .{ }^{13} \mathrm{C} \mathrm{NMR}(75 \mathrm{MHz}$, DMSO- $d_{6}$ ): $\delta$ 170.3, 166.9, 160.8, 149.7 (4CO), 148.1, 144.3 (Ar-C), $141.1(\mathrm{CH}), 139.3,135.8,133.1,130.2$ 128.0, 124.1, 122.6, 120.8, 114.8, $114.3(\mathrm{Ar}-\mathrm{C}), 43.2\left(\mathrm{NCH}_{2} \mathrm{CO}\right), 38.2\left(\mathrm{NCH}_{2} \mathrm{CH}_{3}\right), 12.3\left(\mathrm{CH}_{3}\right)$. Anal. Calcd. For $\mathrm{C}_{21} \mathrm{H}_{20} \mathrm{~N}_{6} \mathrm{O}_{6}$ (452.42): C, 55.75; $\mathrm{H}, 4.46 ; \mathrm{N}, 18.58$; Found $\mathrm{C}, 55.52 ; \mathrm{H}, 4.44 ; \mathrm{N}, 18.71$.

1-Ethyl-2,4-dioxo-(1H,3H)-quinazolin-3-ylacetyl glycyl 3'-methoxy-4-hydroxybenzylidene hydrazone (10d). White crystals $(0.18 \mathrm{~g}, 63 \%), \mathrm{R}_{\mathrm{f}}=0.42\left(\mathrm{~S}_{2}\right), \mathrm{mp} 258-261{ }^{\circ} \mathrm{C} .{ }^{1} \mathrm{H} \mathrm{NMR}\left(300 \mathrm{MHz}, \mathrm{DMSO}-d_{6}\right): \delta 11.28,11.09(1 \mathrm{H}$, 2s, NH), $9.45(1 \mathrm{H}, \mathrm{s}, \mathrm{OH}), 8.63-8.58,8.41-8.37(1 \mathrm{H}, 2 \mathrm{~m}, \mathrm{NH}), 8.07(1 \mathrm{H}, \mathrm{d}, J 7.5 \mathrm{~Hz}, \mathrm{ArH}), 8.10,7.86(1 \mathrm{H}, 2 \mathrm{~s}, \mathrm{CH})$, 7.80-7.75 (1H, t, J 7.8 Hz, ArH), $7.51(1 \mathrm{H}, \mathrm{d}, J 8.4 \mathrm{~Hz}, \mathrm{ArH}), 7.32-7.22(2 \mathrm{H}, \mathrm{m}, \mathrm{ArH}), 7.08-7.02(1 \mathrm{H}, \mathrm{t}, J 8.4 \mathrm{~Hz}$, $\operatorname{ArH}), 6.84-6.78(1 \mathrm{H}, \mathrm{t}, J 8.5 \mathrm{~Hz}, \mathrm{ArH}), 4.66\left(2 \mathrm{H}, \mathrm{s}, \mathrm{NCH}_{2} \mathrm{CO}\right), 4.30-4.25,3.85-3.81\left(2 \mathrm{H}, 2 \mathrm{~m}, \mathrm{NCH}_{2}\right), 4.20-4.10(2 \mathrm{H}$, $\left.\mathrm{m}, \mathrm{NCH}_{2} \mathrm{CH}_{3}\right), 3.81\left(3 \mathrm{H}, \mathrm{s}, \mathrm{OCH}_{3}\right), 1.23-1.18\left(3 \mathrm{H}, \mathrm{t}, J 6.9 \mathrm{~Hz}, \mathrm{NCH}_{2} \mathrm{CH}_{3}\right) .{ }^{13} \mathrm{C} \mathrm{NMR}\left(75 \mathrm{MHz}, \mathrm{DMSO}-d_{6}\right): \delta 169.6$, 166.8, 164.7, 1.60.8 (4CO), 149.7, 148.6 (Ar-C), $143.8(\mathrm{CH}), 139.2,135.4,128.0,125.4,122.6,121.2,115.4$, 114.8, 114.2, $109.1(\mathrm{Ar}-\mathrm{C}), 55.4\left(\mathrm{OCH}_{3}\right), 43.4\left(\mathrm{NCH}_{2} \mathrm{CO}\right), 38.2\left(\mathrm{NCH}_{2} \mathrm{CH}_{3}\right), 12.3\left(\mathrm{CH}_{3}\right)$. Anal. Calcd. For $\mathrm{C}_{22} \mathrm{H}_{23} \mathrm{~N}_{5} \mathrm{O}_{6}$ (453.45): C, 58.27; H, 5.11; N, 15.44; Found C, 58.04; H, 5.20; N, 15.68.

1-Phthalylglycyl-1,3-dicyclohexylurea (11). To a cold solution of $\mathbf{2}(1.17 \mathrm{~g}, 5.0 \mathrm{mmol})$ and phthalylglycine (1.03g, $5.0 \mathrm{mmol})$ and HOBt $(0.68 \mathrm{~g}, 5.0 \mathrm{mmol})$ in methylene chloride $(20.0 \mathrm{ml})$ at $0{ }^{\circ} \mathrm{C}, \mathrm{DCC}(1.15 \mathrm{~g}, 5.5 \mathrm{mmol})$ was added. The reaction mixture was stirred at $0{ }^{\circ} \mathrm{C}$ for $5 \mathrm{~min}$ and at room temperature for $3 \mathrm{hrs}$. The formed precipitate was filtered off and the filtrate was evaporated under reduced pressure. The residue was dissolved in ethyl acetate and washed twice with saturated of $\mathrm{NaCl}$ solution, $5 \% \mathrm{NaHCO}_{3}$ solution and water. After drying over anhydrous sodium sulphate, the solvent was evaporated and the remaining residue was crystallized from ethyl acetate-petroleum ether to yield white crystals of 1-phthalylglycyl-1,3-dicyclohexylurea

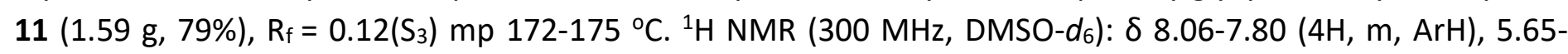
$5.45(1 \mathrm{H}, \mathrm{m}, \mathrm{NH}), 4.31\left(1 \mathrm{H}, \mathrm{s}, \mathrm{NCH}_{2}\right), 3.40-3.25(2 \mathrm{H}, \mathrm{m}, 2 \mathrm{NCH}), 1.75-1.00\left(2 \mathrm{OH}, \mathrm{m}, 10 \mathrm{CH}_{2}\right.$ cyclohexyl rings). Anal. Calcd. For $\mathrm{C}_{23} \mathrm{H}_{29} \mathrm{~N}_{3} \mathrm{O}_{4}$ (411.49): C, 67.13; H, 7.10; N, 10.21; Found C, 67.30; $\mathrm{H}, 7.39 ; \mathrm{N}, 10.36$.

Phthalylglycine 2-(1-ethyl-2,4-dioxo-(1H,3H)-quinazolin-3-yl)ethyl ester (12). To a cold solution $\left(0^{\circ} \mathrm{C}\right)$ of 2 $(1.17 \mathrm{~g}, 5 \mathrm{mmol})$, phthalylglycine $(1.03 \mathrm{~g}, 5 \mathrm{mmol})$ and catalytic amount of DMAP $(0.02 \mathrm{~g}, 0.16 \mathrm{mmol})$ in methylene chloride $(20.0 \mathrm{ml}) \mathrm{DCC}(1.15 \mathrm{~g}, 5.5 \mathrm{mmol})$ was added. The reaction mixture was stirred at $0{ }^{\circ} \mathrm{C}$ for 5 min and at room temperature for $3 \mathrm{hrs}$. The precipitated dicyclohexylurea (DCU) was filtered off and the filtrate was evaporated under reduced pressure. The residue dissolved in methylene chloride and, if necessary, filtered from of any further residual DCU. The solution was washed twice with $0.5 \mathrm{M} \mathrm{HCl}$, saturated solution of $\mathrm{NaHCO}_{3}$ and water. After drying over anhydrous $\mathrm{Na}_{2} \mathrm{SO}_{4}$, the solvent was evaporated and the remaining residue was crystallized from ethyl acetate-petroleum ether to yield white crystals of $12(1.35 \mathrm{~g}, 64 \%), \mathrm{R}_{\mathrm{f}}=$ $0.65\left(\mathrm{~S}_{3}\right) \mathrm{mp} 156-159^{\circ} \mathrm{C} .{ }^{1} \mathrm{H} \mathrm{NMR}\left(300 \mathrm{MHz}, \mathrm{CDCl}_{3}\right): \delta 8.18(1 \mathrm{H}, \mathrm{d}, J 7.8 \mathrm{~Hz}, \mathrm{ArH}), 7.88-7.64(5 \mathrm{H}, \mathrm{m}, \mathrm{ArH}), 7.26-$ $7.19(2 \mathrm{H}, \mathrm{m}, \mathrm{ArH}), 4.49-4.38\left(4 \mathrm{H}, \mathrm{m}, \mathrm{NCH}_{2} \mathrm{CH}_{2} \mathrm{O}\right), 4.41\left(1 \mathrm{H}, \mathrm{s}, \mathrm{NCH}_{2}\right), 4.23-4.16\left(2 \mathrm{H}, \mathrm{m}, \mathrm{NCH}_{2} \mathrm{CH}_{3}\right), 1.37-1.32(3 \mathrm{H}$, $\mathrm{m}, \mathrm{NCH}_{2} \mathrm{CH}_{3}$ ). ${ }^{13} \mathrm{C}$ NMR $\left(75 \mathrm{MHz}, \mathrm{CDCl}_{3}\right.$ ): $\delta$ 167.2, 161.7, 150.4 (CO), 139.6, 135.1, 134.0, 132.0, 129.2, 123.5, 122.7, 115.5, $113.4(\mathrm{Ar}-\mathrm{C}), 62.9\left(\mathrm{NCH}_{2} \mathrm{CH}_{2} \mathrm{O}\right), 40.1\left(\mathrm{NCH}_{2} \mathrm{CH}_{2} \mathrm{O}\right), 38.8\left(\mathrm{NCH}_{2} \mathrm{CH}_{3}\right), 12.5\left(\mathrm{CH}_{3}\right)$. Anal. Calcd. For $\mathrm{C}_{22} \mathrm{H}_{19} \mathrm{~N}_{3} \mathrm{O}_{6}$ (421.40): C, 62.70; $\mathrm{H}, 4.54 ; \mathrm{N}, 9.97$; Found $\mathrm{C}, 63.03 ; \mathrm{H}, 4.85 ; \mathrm{N}, 10.13$.

\section{2-(1-Ethyl-2,4-dioxo-(1H,3H)-quinazolin-3-yl)ethyl acetate (13a)}

Method A. To a cold solution $\left(0^{\circ} \mathrm{C}\right)$ of $2(1.17 \mathrm{~g}, 5 \mathrm{mmol})$, acetic acid $(0.3 \mathrm{~mL}, 5.2 \mathrm{mmol})$ and catalytic amount of DMAP $(0.02 \mathrm{~g}, 0.16 \mathrm{mmol})$ in methylene chloride $(20.0 \mathrm{ml}) \mathrm{DCC}(1.15 \mathrm{~g}, 5.5 \mathrm{mmol})$ was added. Afterward, 
the reaction mixture was treated as described above under the synthesis of the phthalylglycine ester derivative 12 to yield white crystals of $13 a(0.85 \mathrm{~g}, 61 \%), R_{f}=0.64\left(\mathrm{~S}_{3}\right) \mathrm{mp} \mathrm{76-79}{ }^{\circ} \mathrm{C}$.

Method B. A mixture of $2(1.17 \mathrm{~g}, 5 \mathrm{mmol})$, acetic anhydride $(2.0 \mathrm{ml})$ in pyridine $(5.0 \mathrm{ml})$ was stirred at $0{ }^{\circ} \mathrm{C}$ for $1 \mathrm{hr}$. and at room temperature overnight. The reaction mixture was poured into ice, and the solid precipitate was filtered, washed with water and dried in air. Crystallization from ethyl acetate / petroleum ether yielded white crystals of $13 \mathrm{a}(1.06 \mathrm{~g}, 76 \%), \mathrm{R}_{\mathrm{f}}=0.64\left(\mathrm{~S}_{3}\right) \mathrm{mp} 78-80{ }^{\circ} \mathrm{C} .{ }^{1} \mathrm{H} \mathrm{NMR}\left(300 \mathrm{MHz}, \mathrm{CDCl}_{3}\right): \delta 8.18(1 \mathrm{H}, \mathrm{d}, \mathrm{J} 7.8 \mathrm{~Hz}$, $\operatorname{ArH})$, 7.66-7.61 (1H, m, ArH), 7.22- $7.17(2 \mathrm{H}, \mathrm{m}, \mathrm{ArH}), 4.41-4.35\left(4 \mathrm{H}, \mathrm{m}, \mathrm{NCH}_{2} \mathrm{CH}_{2} \mathrm{O}\right), 4.16-4.11(2 \mathrm{H}, \mathrm{m}$, $\left.\mathrm{NCH}_{2} \mathrm{CH}_{3}\right), 1.96\left(3 \mathrm{H}, \mathrm{s}, \mathrm{CH}_{3} \mathrm{CO}\right), 1.33-1.28\left(3 \mathrm{H}, \mathrm{m}, \mathrm{NCH}_{2} \mathrm{CH}_{3}\right) .{ }^{13} \mathrm{C} \mathrm{NMR}\left(75 \mathrm{MHz}, \mathrm{CDCl}_{3}\right): \delta 170.7,161.6,150.3$ (3CO), 139.4, 135.0, 129.0, 122.6, 115.4, $113.2(\mathrm{Ar}-\mathrm{C}), 61.2\left(\mathrm{OCH}_{2}\right), 40.2\left(\mathrm{NCH}_{2}\right), 38.6\left(\mathrm{NCH}_{2} \mathrm{CH}_{3}\right), 20.6\left(\mathrm{CH}_{3}\right)$, $12.3\left(\mathrm{CH}_{3}\right)$. Anal. Calcd. For $\mathrm{C}_{14} \mathrm{H}_{16} \mathrm{~N}_{2} \mathrm{O}_{4}$ (276.29): $\mathrm{C}, 60.86 ; \mathrm{H}, 5.84 ; \mathrm{N}, 10.14$; Found $\mathrm{C}, 60.51 ; \mathrm{H}, 5.80 ; \mathrm{N}, 9.89$.

\section{2-(1-Ethyl-2,4-dioxo-(1H,3H)-quinazolin-3-yl)ethyl benzoate (13b)}

Method A: To a cold solution $\left(0^{\circ} \mathrm{C}\right)$ of $2(1.17 \mathrm{~g}, 5.0 \mathrm{mmol})$, benzoic acid $(0.6 \mathrm{~g}, 5.0 \mathrm{mmol})$ and catalytic amount of DMAP $(0.02 \mathrm{~g}, 0.16 \mathrm{mmol})$ in methylene chloride $(20.0 \mathrm{ml})$ DCC $(1.15 \mathrm{~g}, 5.5 \mathrm{mmol})$ was added. Afterward, the reaction mixture was treated as described above under the synthesis of the phthalylglycine ester derivative 12 to yield white crystals of $12 b(1.1 .16 \mathrm{~g}, 69 \%), R_{f}=0.74\left(S_{3}\right) \mathrm{mp} 129-131{ }^{\circ} \mathrm{C}$.

Method B. To a cold solution of $2(1.17 \mathrm{~g}, 5 \mathrm{mmol})$, in pyridine $(5.0 \mathrm{ml})$ benzoyl chloride $(0.6 \mathrm{ml}, 5 \mathrm{mmol}) \mathrm{was}$ added. The reaction mixture worked up as indicated in the synthesis of 13a (Method B). Crystallization from ethyl acetate / petroleum ether afforded white crystals of $13 \mathrm{~b}(1.35 \mathrm{~g}, 80 \%), \mathrm{R}_{\mathrm{f}}=0.74\left(\mathrm{~S}_{3}\right) \mathrm{mp} 130-133{ }^{\circ} \mathrm{C} .{ }^{1} \mathrm{H}$ NMR $\left(300 \mathrm{MHz}, \mathrm{CDCl}_{3}\right): \delta 8.24(1 \mathrm{H}, \mathrm{d}, J 7.8 \mathrm{~Hz}, \mathrm{ArH}), 8.01(2 \mathrm{H}, \mathrm{d}, J 7.8 \mathrm{~Hz}, \mathrm{ArH}), 7.67-7.19(6 \mathrm{H}, \mathrm{m}, \mathrm{ArH}), 4.63-$ $4.54\left(4 \mathrm{H}, \mathrm{m}, \mathrm{NCH}_{2} \mathrm{CH}_{2} \mathrm{O}\right), 4.21-4.14\left(2 \mathrm{H}, \mathrm{m}, \mathrm{NCH}_{2} \mathrm{CH}_{3}\right), 1.33-1.28\left(3 \mathrm{H}, \mathrm{m}, \mathrm{NCH}_{2} \mathrm{CH}_{3}\right) .{ }^{13} \mathrm{C} \mathrm{NMR}\left(75 \mathrm{MHz}, \mathrm{CDCl}_{3}\right): \delta$ 166.4, 161.7, 150.4 (3CO), 139.6, 135.1, 132.8, 130.0, 129.6, 129.2, 128.2, 122.7, 115.6, $113.4(\operatorname{Ar}-\mathrm{C}), 62.1$ $\left(\mathrm{OCH}_{2}\right), 40.3\left(\mathrm{NCH}_{2}\right), 38.8\left(\mathrm{NCH}_{2} \mathrm{CH}_{3}\right), 12.4\left(\mathrm{CH}_{3}\right)$. Anal. Calcd. For $\mathrm{C}_{19} \mathrm{H}_{18} \mathrm{~N}_{2} \mathrm{O}_{4}(338.36): \mathrm{C}, 67.44 ; \mathrm{H}, 5.36 ; \mathrm{N}$, 8.28; Found $\mathrm{C}, 67.16 ; \mathrm{H}, 5.30 ; \mathrm{N}, 8.36$.

General method for the synthesis of Boc-amino acid 2-(1-ethyl-2,4-dioxo-(1H,3H)-quinazolin-3-yl)ethyl esters $(14 \mathrm{a}-\mathrm{c})$. To a cold solution $\left(0^{\circ} \mathrm{C}\right)$ of $2(1.17 \mathrm{~g}, 5 \mathrm{mmol})$, Boc-amino acid $(5 \mathrm{mmol})$ and catalytic amount of DMAP $(0.02 \mathrm{~g}, 0.16 \mathrm{mmol})$ in dry methylene chloride $(20.0 \mathrm{ml})$ dicyclohexylcarbodiimide $(1.15 \mathrm{~g}, 5.5 \mathrm{mmol})$ was added. The reaction mixture was stirred at $0{ }^{\circ} \mathrm{C}$ for $5 \mathrm{~min}$ and at room temperature for $3 \mathrm{hrs}$. The precipitated DCU was filtered off and the filtrate was evaporated under reduced pressure. The residue was dissolved in methylene chloride and, if necessary, filtered from any further remaining DCU, washed twice with $0.5 \mathrm{M} \mathrm{HCl}$, saturated $\mathrm{NaHCO}_{3}$ solution and water. After drying over anhydrous sodium sulphate, the solvent was evaporated and the remaining residue was precipitated from ethyl acetate by petroleum ether to yield 14a-c.

Boc-glycine 2-(1-ethyl-2,4-dioxo-(1H,3H)-quinazolin-3-yl)ethyl ester (14a). Yellow oil (1.28 $\mathrm{g}, 65 \%), \mathrm{R}_{\mathrm{f}}=$ 0.49(S $\left.\mathrm{S}_{4}\right) .{ }^{1} \mathrm{H}$ NMR $\left(300 \mathrm{MHz}, \mathrm{CDCl}_{3}\right): \delta 8.08(1 \mathrm{H}, \mathrm{d}, J 8.1 \mathrm{~Hz}, \mathrm{ArH}), 7.59-7.54(1 \mathrm{H}, \mathrm{m}, \mathrm{ArH}), 7.13-7.09(2 \mathrm{H}, \mathrm{m}$, ArH), 5.24-5.19 (1H, brs, NH-Boc), 4.35-4.30 (2H, m, NCH $\left.\mathrm{CH}_{2} \mathrm{O}\right), 4.27-4.23\left(2 \mathrm{H}, \mathrm{m}, \mathrm{NCH}_{2} \mathrm{CH}_{2} \mathrm{O}\right), 4.10-4.03(2 \mathrm{H}$, $\left.\mathrm{m}, \mathrm{NCH}_{2} \mathrm{CH}_{3}\right), 3.76-3.74\left(2 \mathrm{H}, \mathrm{m}, \mathrm{NCH}_{2} \mathrm{CO}\right), 1.29\left(9 \mathrm{H}, \mathrm{s}, 3 \mathrm{CH}_{3}\right), 1.25-1.14\left(3 \mathrm{H}, \mathrm{m}, \mathrm{NCH}_{2} \mathrm{CH}_{3}\right) .{ }^{13} \mathrm{C} \mathrm{NMR}(75 \mathrm{MHz}$, $\mathrm{CDCl}_{3}$ ): $\delta 169.9,161.4,155.4,150.1$ (4CO), 139.2, 134.9, 128.7, 122.4, 115.1, 113.1 (Ar-C), 79.2 (O-C, t-butyl), $61.9\left(\mathrm{NCH}_{2} \mathrm{CH}_{2} \mathrm{O}\right), 42.1\left(\mathrm{NCH}_{2} \mathrm{CO}\right), 39.9\left(\mathrm{NCH}_{2} \mathrm{CH}_{2} \mathrm{O}\right), 38.5\left(\mathrm{NCH}_{2} \mathrm{CH}_{3}\right), 27.9\left(3 \mathrm{CH}_{3}, t\right.$-butyl), $12.2\left(\mathrm{CH}_{3}\right)$. Anal. Calcd. For $\mathrm{C}_{19} \mathrm{H}_{25} \mathrm{~N}_{3} \mathrm{O}_{6}$ (391.42): C, 58.30; $\mathrm{H}, 6.44 ; \mathrm{N}, 10.74$; Found $\mathrm{C}, 58.11 ; \mathrm{H}, 6.24 ; \mathrm{N}, 10.97$.

Boc-L-leucine 2-(1-ethyl-2,4-dioxo-(1H,3H)-quinazolin-3-yl)ethyl ester (14b). Yellow oil $(1.51 \mathrm{~g}, 67 \%), R_{f}=$ 0.74(S4). ${ }^{1} \mathrm{H}$ NMR (300 MHz, CDCl 3$): \delta 8.07(1 \mathrm{H}, \mathrm{d}, J 7.8 \mathrm{~Hz}, \mathrm{ArH}), 7.58-7.53(1 \mathrm{H}, \mathrm{m}, \operatorname{ArH}), 7.12-7.07(2 \mathrm{H}, \mathrm{m}$, ArH), 5.07-5.01 (1H, brs, NH-Boc), 4.34-4.20 (4H, m, NCH$\left.{ }_{2} \mathrm{CH}_{2} \mathrm{O}\right), 4.18-4.03\left(3 \mathrm{H}, \mathrm{m}, \mathrm{NCH}, \mathrm{NCH}_{2} \mathrm{CH}_{3}\right), 1.59-1.33$ $\left(3 \mathrm{H}, \mathrm{m}, \mathrm{CH}_{2}, \mathrm{CH}\right), 1.26\left(9 \mathrm{H}, \mathrm{s}, 3 \mathrm{CH}_{3}\right), 1.24-1.14\left(3 \mathrm{H}, \mathrm{m}, \mathrm{NCH}_{2} \mathrm{CH}_{3}\right), 0.80\left(6 \mathrm{H}, \mathrm{d}, J 6.3 \mathrm{~Hz}, 2 \mathrm{CH}_{3}\right) .{ }^{13} \mathrm{C} \mathrm{NMR}(75 \mathrm{MHz}$, $\mathrm{CDCl}_{3}$ ): $\delta 172.6,161.3,155.0,149.9$ (4CO), 139.2, 134.8, 128.7, 122.3, 115.1, 113.1 (Ar-C), 78.9 (O-C, t-butyl), 
$61.7\left(\mathrm{NCH}_{2} \mathrm{CH}_{2} \mathrm{O}\right), 51.4(\mathrm{NCH}), 41.0\left(\mathrm{NCH}_{2} \mathrm{CH}_{2} \mathrm{O}\right), 39.8\left(\mathrm{NCH}_{2} \mathrm{CH}_{3}\right), 38.4\left(\mathrm{CH}_{2}\right), 27.8\left(3 \mathrm{CH}_{3}, t\right.$-butyl), $24.2(\mathrm{CH})$, $22.5\left(\mathrm{CH}_{3}\right), 21.5\left(\mathrm{CH}_{3}\right), 12.1\left(\mathrm{CH}_{3}\right)$. Anal. Calcd. For $\mathrm{C}_{23} \mathrm{H}_{33} \mathrm{~N}_{3} \mathrm{O}_{6}(447.52): \mathrm{C}, 61.73 ; \mathrm{H}, 7.43 ; \mathrm{N}, 9.39 ;$ Found $\mathrm{C}$, $61.51 ; \mathrm{H}, 7.35 ; \mathrm{N}, 9.62$.

Boc-L-tryptophane 2-(1-ethyl-2,4-dioxo-(1H,3H)-quinazolin-3-yl)ethyl ester (14c). White crystals (1.68 g, $63 \%), \mathrm{R}_{\mathrm{f}}=0.41\left(\mathrm{~S}_{4}\right) \mathrm{mp} 138-141{ }^{\circ} \mathrm{C} .{ }^{1} \mathrm{H} \mathrm{NMR}\left(300 \mathrm{MHz}, \mathrm{CDCl}_{3}\right): \delta 8.25(1 \mathrm{H}, \mathrm{d}, J 7.8 \mathrm{~Hz}, \mathrm{ArH}), 7.96(1 \mathrm{H}, \mathrm{brs}, \mathrm{Ind}-$ $\mathrm{NH})$, 7.71- $7.66(1 \mathrm{H}, \mathrm{t}, J 7.8 \mathrm{~Hz}, \mathrm{ArH}), 7.56(1 \mathrm{H}, \mathrm{d}, J 7.8 \mathrm{~Hz}, \mathrm{ArH}), 7.27-7.04(5 \mathrm{H}, \mathrm{m}, \mathrm{ArH}), 6.99(1 \mathrm{H}, \mathrm{s}, \mathrm{Ind}-2-\mathrm{H})$, 5.20-5.13 (1H, brs, NH-Boc), $4.62(1 \mathrm{H}, \mathrm{m}, \mathrm{NCH}), 4.40-4.34\left(4 \mathrm{H}, \mathrm{m}, \mathrm{NCH}_{2} \mathrm{CH}_{2} \mathrm{O}\right), 4.16-4.10\left(2 \mathrm{H}, \mathrm{m}, \mathrm{NCH}_{2} \mathrm{CH}_{3}\right)$, 3.09-3.05 (2H, m, CH 2$), 1.39\left(9 \mathrm{H}, \mathrm{s}, 3 \mathrm{CH}_{3}\right), 1.35-1.26\left(3 \mathrm{H}, \mathrm{m}, \mathrm{NCH}_{2} \mathrm{CH}_{3}\right) .{ }^{13} \mathrm{C} \mathrm{NMR}\left(75 \mathrm{MHz}, \mathrm{CDCl}_{3}\right): \delta 171.8$, 161.7, 155.1, 150.4 (4CO), 139.5, 135.1, 129.1, 122.8, 121.8, 119.3, 118.6, 115.5, 113.4, 111.0 (Ar-C), 79.5 (O-C, t-butyl), $62.3\left(\mathrm{NCH}_{2} \mathrm{CH}_{2} \mathrm{O}\right), 54.3(\mathrm{NCH}), 40.1\left(\mathrm{NCH}_{2} \mathrm{CH}_{2} \mathrm{O}\right), 38.8\left(\mathrm{NCH}_{2} \mathrm{CH}_{3}\right), 28.2\left(3 \mathrm{CH}_{3}, t\right.$-butyl), $12.4\left(\mathrm{CH}_{3}\right)$. Anal. Calcd. For $\mathrm{C}_{28} \mathrm{H}_{32} \mathrm{~N}_{4} \mathrm{O}_{6}$ (520.58): C, 64.60; $\mathrm{H}, 6.20 ; \mathrm{N}, 10.76$; Found $\mathrm{C}, 64.48 ; \mathrm{H}, 6.24 ; \mathrm{N}, 10.91$.

General method for the synthesis of amino acid 2-(1-ethyl-2,4-dioxo-(1H,3H)-quinazolin-3-yl)ethyl ester hydrochloridse (15a-c). Boc-amino acid ester derivative 14a-c $(1.5 \mathrm{mmol})$ was stirred in $1 \mathrm{M} \mathrm{HCl}$ in ethyl acetate $(15 \mathrm{~mL})$ for $2 \mathrm{hrs}$. at room temperature. Afterward, the reaction mixture was concentrated and left to cool. The formed precipitate was filtered off, washed with dry ethyl acetate and dried.

Glycine 2-(1-ethyl-2,4-dioxo-(1H,3H)-quinazolin-3-yl)ethyl ester hydrochloride (15a). White crystals (0.32 g, $63 \%), R_{f}=0.40\left(S_{2}\right), m p 184-186{ }^{\circ} \mathrm{C} .{ }^{1} \mathrm{H} N M R\left(300 \mathrm{MHz}, \mathrm{DMSO}-d_{6}\right): \delta 8.90-8.50\left(3 \mathrm{H}, \mathrm{brs}, \mathrm{NH}_{3}{ }^{+}\right), 8.03(1 \mathrm{H}, \mathrm{d}, J 7.8$ $\mathrm{Hz}, \mathrm{ArH}), 7.78-7.72(1 \mathrm{H}, \mathrm{t}, J 7.9 \mathrm{~Hz}, \mathrm{ArH}), 7.49(1 \mathrm{H}, \mathrm{d}, J 8.4 \mathrm{~Hz}, \mathrm{ArH}), 7.29-7.24(1 \mathrm{H}, \mathrm{t}, J \mathrm{~J} .6 \mathrm{~Hz}, \mathrm{ArH}), 4.40-4.36$ $\left(2 \mathrm{H}, \mathrm{m}, \mathrm{NCH}_{2} \mathrm{CH}_{2} \mathrm{O}\right), 4.23-4.20\left(2 \mathrm{H}, \mathrm{m}, \mathrm{NCH}_{2} \mathrm{CH}_{2} \mathrm{O}\right), 4.14-4.07\left(2 \mathrm{H}, \mathrm{m}, \mathrm{NCH}_{2} \mathrm{CH}_{3}\right), 3.65\left(2 \mathrm{H}, \mathrm{s}, \mathrm{NCH}_{2} \mathrm{CO}\right), 1.25-1.16$ $\left(3 \mathrm{H}, \mathrm{t}, J 6.9 \mathrm{~Hz}, \mathrm{NCH}_{2} \mathrm{CH}_{3}\right) .{ }^{13} \mathrm{C} \mathrm{NMR}\left(75 \mathrm{MHz}, \mathrm{DMSO}-d_{6}\right): \delta$ 167.4, 161.2, 149.9 (3CO), 139.2, 135.5, 128.0, 122.6, 114.8, $114.3(\mathrm{Ar}-\mathrm{C}), 62.2\left(\mathrm{OCH}_{2}\right), 38.3\left(\mathrm{NCH}_{2} \mathrm{CH}_{3}\right), 12.3\left(\mathrm{CH}_{3}\right)$. Anal. Calcd. For $\mathrm{C}_{14} \mathrm{H}_{18} \mathrm{~N}_{3} \mathrm{O}_{4} \mathrm{Cl}(327.76): \mathrm{C}$, 51.30; H, 5.54; N, 12.82; Found C, 51.56; H, 5.62; N, 12.59.

L-Leucine 2-(1-ethyl-2,4-dioxo-(1H,3H)-quinazolin-3-yl)ethyl ester hydrochloride (15b). White crystals (0.25 g, 43\%), $R_{f}=0.56\left(\mathrm{~S}_{2}\right), \mathrm{mp} 177-180{ }^{\circ} \mathrm{C} .{ }^{1} \mathrm{H} N M R\left(300 \mathrm{MHz}, \mathrm{CDCl}_{3}\right): \delta 8.90-8.70\left(3 \mathrm{H}, \mathrm{brs}, \mathrm{NH}_{3}{ }^{+}\right), 8.16(1 \mathrm{H}, \mathrm{d}, J 7.8 \mathrm{~Hz}$, $\mathrm{ArH})$, 7.66- $7.60(1 \mathrm{H}, \mathrm{t}, J 7.9 \mathrm{~Hz}, \mathrm{ArH}), 7.27-7.16(2 \mathrm{H}, \mathrm{m}, \mathrm{ArH}), 4.52-4.35\left(4 \mathrm{H}, \mathrm{m}, \mathrm{NCH}_{2} \mathrm{CH}_{2} \mathrm{O}\right), 4.18-4.09(2 \mathrm{H}, \mathrm{m}$, $\left.\mathrm{NCH}_{2} \mathrm{CH}_{3}\right), 4.01-3.97(1 \mathrm{H}, \mathrm{m}, \mathrm{NCHCO}), 1.91-1.72\left(3 \mathrm{H}, \mathrm{m}, \mathrm{CH}_{2}, \mathrm{CH}\right), 1.32-1.27\left(3 \mathrm{H}, \mathrm{t}, \mathrm{J} 7.0 \mathrm{~Hz}, \mathrm{NCH}_{2} \mathrm{CH}_{3}\right), 0.86$ $\left(6 \mathrm{H}, \mathrm{d}, \mathrm{J} 5.7 \mathrm{~Hz}, 2 \mathrm{CH}_{3}\right) .{ }^{13} \mathrm{C} \mathrm{NMR}\left(75 \mathrm{MHz} \mathrm{CDCl}_{3}\right): \delta 169.4,161.6,150.2(3 \mathrm{CO}), 139.4,135.1,129.1,122.7,115.3$, 113.4 (Ar-C), $63.2\left(\mathrm{NCH}_{2} \mathrm{CH}_{2} \mathrm{O}\right), 51.6(\mathrm{NCH}), 39.9\left(\mathrm{NCH}_{2} \mathrm{CH}_{2} \mathrm{O}\right), 39.0\left(\mathrm{NCH}_{2} \mathrm{CH}_{3}\right), 38.8\left(\mathrm{CH}_{2}\right), 24.2(\mathrm{CH}), 22.1\left(\mathrm{CH}_{3}\right)$, $21.7\left(\mathrm{CH}_{3}\right), 12.4\left(\mathrm{CH}_{3}\right)$. Anal. Calcd. For $\mathrm{C}_{18} \mathrm{H}_{26} \mathrm{~N}_{3} \mathrm{O}_{4} \mathrm{Cl}$ (383.87): C, 56.32; $\mathrm{H}, 6.83 ; \mathrm{N}, 10.95 ;$ Found $\mathrm{C}, 56.47 ; \mathrm{H}$, 6.90; N, 10.74.

L-Tryptophane 2-(1-ethyl-2,4-dioxo-(1H,3H)-quinazolin-3-yl)ethyl ester hydrochloride (15c). White crystals $(0.52 \mathrm{~g}, 74 \%), \mathrm{R}_{\mathrm{f}}=0.47\left(\mathrm{~S}_{2}\right), \mathrm{mp}(\mathrm{dec}) 146-149{ }^{\circ} \mathrm{C} .{ }^{1} \mathrm{H} \mathrm{NMR}\left(300 \mathrm{MHz}, \mathrm{CDCl}_{3}\right): \delta 9.68(1 \mathrm{H}, \mathrm{brs}$, Ind-NH), 8.50-8.20 $\left(3 \mathrm{H}\right.$, brs, $\left.\mathrm{NH}_{3}{ }^{+}\right), 8.04(1 \mathrm{H}, \mathrm{d}, J 7.8 \mathrm{~Hz}, \mathrm{ArH}), 7.55-6.75(8 \mathrm{H}, \mathrm{m}, 7 \mathrm{ArH}$, Ind-2-H), 4.17-3.90 $(7 \mathrm{H}, \mathrm{m}, \mathrm{NCH}$, $\left.\mathrm{NCH}_{2} \mathrm{CH}_{2} \mathrm{O}, \mathrm{NCH}_{2} \mathrm{CH}_{3}\right), 3.50-3.20\left(2 \mathrm{H}, \mathrm{m}, \mathrm{CH}_{2}\right), 1.25-1.18\left(3 \mathrm{H}, \mathrm{m}, \mathrm{NCH}_{2} \mathrm{CH}_{3}\right) .{ }^{13} \mathrm{C} \mathrm{NMR}\left(75 \mathrm{MHz}, \mathrm{CDCl}_{3}\right): \delta 169.0$, 161.7, 150.1 (3CO), 139.2, 136.1, 135.2, 128.9, 126.5, 126.1, 122.9, 121.3, 118.8, 117.6, 115.1, 113.4, 111.7, 105.7 (Ar-C), $63.5\left(\mathrm{NCH}_{2} \mathrm{CH}_{2} \mathrm{O}\right), 53.4(\mathrm{NCH}), 39.8\left(\mathrm{NCH}_{2} \mathrm{CH}_{2} \mathrm{O}\right), 38.8\left(\mathrm{NCH}_{2} \mathrm{CH}_{3}\right), 25.7\left(\mathrm{CH}_{2}\right), 12.4\left(\mathrm{CH}_{3}\right)$. Anal. Calcd. For $\mathrm{C}_{23} \mathrm{H}_{25} \mathrm{~N}_{4} \mathrm{O}_{4} \mathrm{Cl}$ (456.92): C, 60.46; H, 5.51; N, 12.26; Found C, 60.68; H, 5.63; N, 12.03.

In vitro antiproliferative activity. First, a preliminary investigation of antitumor activity of the synthesized compounds were performed at $100 \mu \mathrm{g} / \mathrm{mL}$. Based upon these study the inactive compounds were excluded from further investigation. Cytotoxicity of the newly synthesized compounds was tested against human liver carcinoma cell line (HepG2) using the method of Skehan et al. ${ }^{32}$ 


\section{Supplementary Material}

Synthesis of the starting compounds 1 and 2 and copies of ${ }^{1} \mathrm{H}$ and ${ }^{13} \mathrm{C}$ NMR spectra of the new compounds can be found in the supplementary material file.

\section{References}

1. Rang, H. H.; Dale, M. M.; Ritter, J. M.; Flower, R. J.; Henderson, G. Rang and Dale's Pharmacology. 7th ed. London: Elsevier; 2012: 673. https://doi.org/10.1016/B978-0-7020-3471-8.00055-X

2. Jemal, A.; Bray, F.; Center, M. M.; Ferlay, J.; Ward, E.; Forman, D. CA Cancer J. Clin. 2011, 61, 69. https://doi.org/10.3322/caac.20107

3. Antoni, S.; Soerjomataram, I.; Møller, B.; Braya, F.; Ferlay, J. Bull. World Health Organ. 2016, $94,184$. https://doi.org/10.2471/BLT.15.164384

4. DeVita,V. T. Jr. Chu, E. Cancer Res. 2008, 68, 8643. https://doi.org/10.1158/0008-5472.CAN-07-6611

5. Chari, R. V. J.; Miller, M. L.; Widdison, W. C. Angew. Chem. Int. Ed. 2014, 53, 3796. https://doi.org/10.1002/anie.201307628

6. Reddy, T. S.; Reddy, V. G.; Kulhari, H.; Shukla, R.; Kamal, A.; Bansal, V. Eur. J. Med. Chem. 2016, $117,157$. https://doi.org/10.1016/i.ejmech.2016.03.051

7. Khan, I.; Zaib, S.; Batool, S.; Abbas, N.; Ashraf, Z.; Iqbal, J.; Saeed, A. Bioorg. Med. Chem. 2016, $24,2361$. https://doi.org/10.1016/i.bmc.2016.03.031

8. Mowafy, S.; Galanis, A.; Doctor, Z. M.; Paranal, R. M.; Lasheen, D. S.; Farag, N. A.; Jänne, P. A.; Abouzid, K. A. M. Bioorg. Med. Chem. 2016, 24, 3501. https://doi.org/10.1016/i.bmc.2016.05.063

9. Qin, X.; Li, Z.; Yang, L.; Liu, P.; Hu, L.; Zeng, C.; Pan, Z. Bioorg. Med. Chem. 2016, 24, 2871. https://doi.org/10.1016/i.bmc.2016.01.003

10. Fiorito, S.; Taddeo, V. A.; Epifano, F.; Genovese, S. Arkivoc 2017, ii, 68.

11. El Fekki, I.; Ali, I. A. I.; Fathalla, W.; Alsheikh, A. A.; El Tamneya, E. Arkivoc 2017, iv, 104.

12. Spanò, V.; Montalbano, A.; Carbone, A.; Parrino, B.; Diana, P.; Cirrincione, G.; Castagliuolo, I.; Brun, P.; Issinger, O. G.; Tisi, S.; Prima, I.; Vedaldi, D.; Salvador, A.; Barraja, P. Eur. J. Med. Chem. 2014, 74, 340. https://doi.org/10.1016/i.ejmech.2013.10.014

13. Barraja, P.; Spanò, V.; Diana, P.; Carbone, A.; Cirrincione, G. Tetrahedron Lett. 2009, 50, 5389. https://doi.org/10.1016/i.tetlet.2009.07.045

14. Muhsin, M.; Graham, J.; Kirkpatrick, P. Nat. Rev. Drug Discov. 2003, 2, 515. https://doi.org/10.1038/nrd1136

15. Dowell, J.; Minna, J. D.; Kirkpatrick, P. Nat. Rev. Drug Discov. 2005, 4, 13. https://doi.org/10.1038/nrd1612

16. Moy, B.; Kirkpatrick, P.; Kar, S; Goss, P. Nat. Rev. Drug Discov. 2007, 6, 431. https://doi.org/10.1038/nrd2332

17. Pui, C. -H.; Jeha, S. Nat. Rev. Drug Discov. 2007, 6, 149. https://doi.org/10.1038/nrd2240

18. Marzaro, G.; Guiotto, A.; Chilin, A. Expert Opin. Ther. Patents 2012, 22, 223. 
19. Galm, U.; Hager, M. H.; Lanen, S. G. V.; Ju, J.; Thorson, J. S.; Shen, B. Chem. Rev. 2005, 105, 739. https://doi.org/10.1021/cr030117g

20. Lo, Y. -S.; Tseng, W. -H.; Chuang, C. -Y.; Hou, M. -H. Nucleic Acids Res. 2013, 7, 4284. https://doi.org/10.1093/nar/gkt084

21. Vicini, P.; Incerti, M.; Doytchinova, I. A.; Colla, P. L.; Busonera, B.; Loddo, R. Eur. J. Med. Chem. 2006, 41, 624.

https://doi.org/10.1016/j.ejmech.2006.01.010

22. Kumar, D.; Kumar, N. M.; Ghosh, S.; Shah, K. Bioorg. Med. Chem. Lett. 2012, 22, 212. https://doi.org/10.1016/j.bmcl.2011.11.031

23. Nasr, T.; Bondock, S.; Youns, M. Eur. J. Med. Chem. 2014, 76, 539. https://doi.org/10.1016/i.ejmech.2014.02.026

24. Tweedie, D. J.; Erikson, J. M.; Prough, R. A. Pharmac. Ther. 1987, 34, 127. https://doi.org/10.1016/0163-7258(87)90095-7

25. Ukrainets, I. V.; Kravtsova, V. V.; Tkach, A. A.; Mamchur, V. I.; Kovalenko, E. Yu. Chem. Heterocycl. Comp. 2010, 46, 96.

https://doi.org/10.1007/s10593-010-0475-4

26. Smith, P. A. S. Org. React. 1946, 3, 337.

27. Montalbetti, C. A. G. N.; Falque, V. Tetrahedron 2005, 61, 10827. https://doi.org/10.1016/i.tet.2005.08.031

28. Ozdemir, A.; Turan-Zitounil, G.; Kaplancikli, Z. A.; Altintop, M. D. J. Serb. Chem. Soc. 2012, 77, 141. https://doi.org/10.2298/JSC1103211710

29. Mohammed, A. F.; Hussein, M. A.; Abdel-Moty, S. G.; Abdel-Alim, A. M. Bull. Pharm. Sci., Assiut Univ. 2011, 34, 77.

30. Bayrak, H.; Demirbas, A.; Karaoglu, S. A.; Demirbas, N. Eur. J. Med. Chem. 2009, 44, 1057. https://doi.org/10.1016/i.ejmech.2008.06.019

31. Uygun, Y.; Bayrak, H.; Ozkan, H. Turk. J. Chem. 2013, 37, 812. https://doi.org/10.3906/kim-1212-66

32. Skehan, P.; Storeng, R.; Scudiero, D.; Monks, A.; McMahon, J.; Vistica, D.; Warren, J. T.; Bokesch, H.; Kenney, S.; Boyd, M. R. J. Natl. Cancer Inst. 1990, 82, 1107. https://doi.org/10.1093/jnci/82.13.1107 